British Journal of Nutrition (2022), 127, 298-317

doi:10.1017/S0007114521000921

(C) The Author(s), 2021. Published by Cambridge University Press on behalf of The Nutrition Society. This is an Open Access article, distributed under the terms of the Creative Commons Attribution-NonCommercial-ShareAlike licence (http://creativecommons.org/licenses/by-nc-sa/4.0/) which permits non-commercial re-use, distribution, and reproduction in any medium, provided the same Creative Commons licence is included and the original work is properly cited. The written permission of Cambridge University Press must be obtained for commercial re-use.

\title{
Systematic review of the effects of pandemic confinements on body weight and their determinants
}

\author{
Moien AB Khan ${ }^{1,2 *}$, Preetha Menon ${ }^{3}$, Romona Govender ${ }^{1}$, Amal MB Abu Samra ${ }^{1}$,
} Kholoud K Allaham ${ }^{1}$, Javaid Nauman ${ }^{3,4,5}$, Linda Östlundh ${ }^{6}$, Halla Mustafa ${ }^{1}$, Jane E.M. Smith ${ }^{7}$ and Juma M AlKaabi ${ }^{8}$

${ }^{1}$ Nutrition Studies Research Group, Department of Family Medicine, College of Medicine and Health Sciences, United Arab Emirates University, Al-Ain, United Arab Emirates

${ }^{2}$ Primary Care, NHS North West London, London TW3 3EB, UK

${ }^{3}$ Institute of Public Health, College of Medicine and Health Sciences, United Arab Emirates University, Al-Ain, United Arab Emirates

${ }^{4}$ Department of Circulation and Medical Imaging, Faculty of Medicine and Health Sciences, Norwegian University of Science and Technology, Trondheim, Norway

${ }^{5}$ Healthy Living for Pandemic Event Protection (HL - PIVOT) Network, Chicago, IL, USA

${ }^{6}$ National Medical Library, College of Medicine and Health Sciences, United Arab Emirates University, Al-Ain, United Arab Emirates

${ }^{7}$ Bodreinallt Surgery, NHS Wales, Conwy, LL32 8AT, UK

${ }^{8}$ Department of Internal Medicine, College of Medicine and Health Sciences, United Arab Emirates University, Al-Ain, United Arab Emirates

(Submitted 16 September 2020 - Final revision received 3 March 2021 - Accepted 7 March 2021 - First published online 12 March 2021)

\section{Abstract}

Pandemics and subsequent lifestyle restrictions such as 'lockdowns' may have unintended consequences, including alterations in body weight. This systematic review assesses the impact of pandemic confinement on body weight and identifies contributory factors. A comprehensive literature search was performed in seven electronic databases and in grey sources from their inception until 1 July 2020 with an update in PubMed and Scopus on 1 February 2021. In total, 2361 unique records were retrieved, of which forty-one studies were identified eligible: one case-control study, fourteen cohort and twenty-six cross-sectional studies (469, 362 total participants). The participants ranged in age from 6 to 86 years. The proportion of female participants ranged from $37 \%$ to $100 \%$. Pandemic confinements were associated with weight gain in $7 \cdot 2-72.4 \%$ of participants and weight loss in $11 \cdot 1-32.0 \%$ of participants. Weight gain ranged from 0.6 (sD 1.3 ) to 3.0 (sD 2.4 ) $\mathrm{kg}$, and weight loss ranged from 2.0 (SD 1.4) to 2.9 (SD 1.5) kg. Weight gain occurred predominantly in participants who were already overweight or obese. Associated factors included increased consumption of unhealthy food with changes in physical activity and altered sleep patterns. Weight loss during the pandemic was observed in individuals with previous low weight, and those who ate less and were more physically active before lockdown. Maintaining a stable weight was more difficult in populations with reduced income, particularly in individuals with lower educational attainment. The findings of this systematic review highlight the short-term effects of pandemic confinements.

Key words: Quarantine: Lockdown: Body weight: Obesity: Weight determinants: Pandemic

Devastating physical morbidity and mortality outcomes due to coronavirus disease 2019 (COVID-19) have been mitigated by $^{(1,2)}$ social distancing and quarantine measures ${ }^{(3)}$, with significant direct and indirect health implications. Although lockdown has reduced the 'R number', physical well-being may have suffered from increased levels of stress, anxiety and mental health issues $^{(4-6)}$. Moderate weight gain in people with a normal BMI has an adverse effect on metabolism, which increases the risk of diabetes, $\mathrm{CVD}^{(7)}$ or long-term ill-health ${ }^{(8)}$. Lockdown may precipitate weight gain similar to that seen during the 6-week summer holidays because of increased inactive time spent at home and snacking on energy-dense foods ${ }^{(9-11)}$. Rundle and

Abbreviation: COVID-19, coronavirus disease 2019.

* Corresponding author: Dr Moien AB Khan, email moien.khan@uaeu.ac.ae 
colleagues argued that the extent and haste of the restrictions have exaggerated these observations ${ }^{(12)}$ leading to rapid weight gain. This presents particular issues with the gained weight being more difficult to shed ${ }^{(13)}$. Moreover, physical and social isolation is a recognised risk factor for obesity ${ }^{(14)}$, with weight due to overconsumption, particularly when large "emergency" food stores are present ${ }^{(15)}$. Reduced physical activity has further exacerbated the weight gain.

The COVID-19 outbreak adversely affected food supply and demand on a global scale ${ }^{(16)}$. For some, lockdown gave more time to cook and overconsume, while those who were financially disadvantaged suffered from malnutrition and weight loss because of inflated food prices and food insecurity ${ }^{(17,18)}$.

Recent research has linked obesity to an increased risk of contracting severe infections of COVID-19, thereby increasing the risk for extended hospitalisation and increased mortality ${ }^{(19)}$. Importantly, therapeutic interventions and prophylactic treatments are more difficult and less effective in this group ${ }^{(20-25)}$, with resultant poorer outcomes. Thus, weight gain secondary to pandemic confinement has an increased significance.

As the pandemic unfolds, researchers all over the globe try to better understand the prevalence, factors involved and impact of weight change in order to guide prevention strategies that will address this major public health crisis. These efforts have led to the identification of multiple determinants including biological, psychological and sociological processes that influence body weight during the pandemic. In this report, the interplay between these factors has been extrapolated from a systematic review of the current literature. Through an analysis of these observations, future public health interventions can be determined.

\section{Materials and methods}

\section{Methods and analysis}

This review has been informed by the Cochrane Handbook for Systematic Reviews of Interventions ${ }^{(26)}$ and is reported in accordance with the Preferred Reporting Items for Systematic Reviews and Meta-analyses ${ }^{(27)}$. The review protocol is registered in the PROSPERO International Register of Systematic Reviews (Registration number CRD42020193440). This systematic review did not need approval from the ethics committee or required informed consent from the study populations as the data were retrieved from open-source databases and internet searches.

\section{Search strategy}

A medical librarian (L.Ö.) performed a comprehensive literature search in the electronic databases PubMed Embase, Scopus, PsycInfo, Cochrane, CINAHL and Web of Science in June and July of 2020. Search terms related to 'pandemics' AND 'body weight' AND 'confinement' were systematically developed with the help of PubMed and PubMed's MeSH and reviewed and discussed with a subject specialist (M.A.B.K.). The search string developed in PubMed was later adapted and applied to all databases. A combination of the search fields of 'Title', 'Abstract' and $\mathrm{MeSH} /$ Thesaurus (when available) was used to ensure the best possible search precision and results. No filters or limitations were applied to ensure the inclusion of pre-indexed materials.
All databases were searched from their inception until July 2020. Selected sources of grey literature and the preprint archive medRxiv were additionally included in the literature search. A search update in PubMed and Scopus was conducted on 1 February 2021. No additional relevant studies were located after hand screening the results from the updated search.

A search log with database specifications, detailed search strings, results and notes for all sources included in the search is available in online Supplementary Appendix 1.

\section{Inclusion and exclusion criteria}

All study designs relevant to human pandemic confinements and their effects on body weight were included (Table 1). All age groups were included, and there were no language restrictions.

This review was extended to articles published from the time of inception until 1 July 2020 and from an update in PubMed and Scopus on 1 February 2021. The primary outcome was to determine the effects of pandemic confinements on body weight. The secondary research outcome was to identify factors affecting body weight during pandemic confinements.

We excluded animal studies and studies investigating the effect of obesity or overweight on various outcomes during the pandemic. We also excluded studies that only narrated the effects of obesity or overweight as a risk factor worsening pandemic-related disease. Studies on diseases, such as HIV, measles and mumps, were also excluded.

\section{Screening and selection}

All references identified in the databases and grey searches ( $n$ 5070) were uploaded to the systematic review tool Covidence (Veritas Health Innovation, 2020) for automatic deduplication and blinded screening (Preferred Reporting Items for Systematic Reviews and Meta-analyses flow diagram (Fig. 1)). Two reviewers (H.M. and M.A.B.K.) independently screened the references at both the title/abstract ( $n$ 2361) and full-text level ( $n$ 78). A third reviewer (P.M.) resolved any conflicts. The grey references and preprints were screened and deduplicated manually by M.A.B.K. and L.Ö. Finally, the reference lists of the included papers were hand screened. Those full-text articles that did not meet the inclusion criterion were excluded ( $n 27$ ) (Fig. 1). One study investigating weight gain exclusively in pregnant women was excluded ${ }^{(28)}$ as it was impossible to distinguish physiological from pandemic-related weight gain in this group.

\section{Data extraction}

The study characteristics including the authors, year of publication, country of origin, study design, research instruments used, validity of survey questionnaire, proportion of female participants, age range and mean age of participants, mean BMI of participants and mean weight of participants were extracted by one reviewer (M.A.B.K.). The other reviewers (P.M., R.G. and A.M.B.A.S.) extracted and reviewed the data independently. Determinants that had an impact on body weight were extracted and reviewed (primarily by M.A.B.K. and secondarily by P.M., R.G. and A.M.B.A.S.). 
Table 1. Inclusion and exclusion criteria

\begin{tabular}{|c|c|c|}
\hline & Inclusion & Exclusion \\
\hline Population & Human studies on pandemic confinement & $\begin{array}{l}\text { Animal studies } \\
\text { Studies investigating the effect of obesity or overweight } \\
\text { on various outcomes during the pandemics } \\
\text { Diseases such as HIV, measles and mumps }\end{array}$ \\
\hline Effect & $\begin{array}{l}\text { Studies describing the impact of quarantine on body weight } \\
\text { Studies showing the impact of quarantine on the overweight/obese } \\
\text { population }\end{array}$ & $\begin{array}{l}\text { Studies showing obesity or overweight as a risk factor } \\
\text { for the pandemic }\end{array}$ \\
\hline Study & $\begin{array}{l}\text { Designs: all study designs. Language: all languages. } \\
\text { Year: publication year inception - } 1 \text { February } 2021\end{array}$ & \\
\hline
\end{tabular}

Records identified in electronic databases: 2490

(568 in PubMed , 644 in Embase, 91 in Cochrane, 423 in Web of Science, 673 in Scopus, 20 in PsycInfo and 71 in CINAHL)
Additional records identified in gray sources and preprints: 626

(588 in medRxiv, 12 in ClinicalTrials.gov, 4 in NY

Academy of Medicine- Grey Literature Report, 12 in Bielefeld Academic Search Engine and 10 in Open Grey)

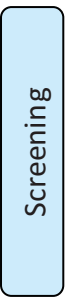

Records identified in the updated PubMed and Scopus search: 1954*

(934 in PubMed and 1020 in Scopus)

*No search date restrictions were applied to avoid missing any backfile materials in the update.

Total number of records identified: 5070

Number of duplicates removed: 2709

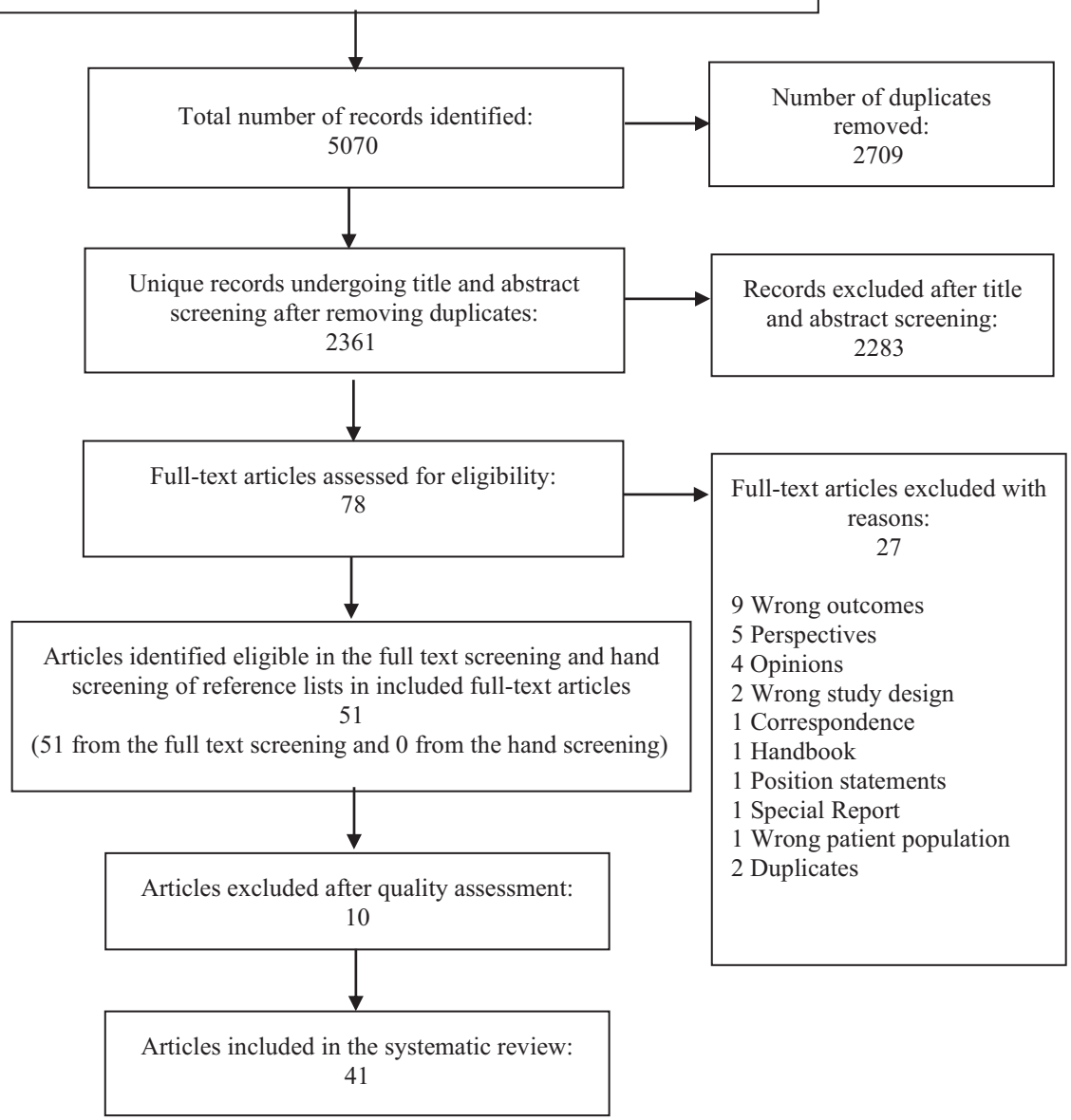

Fig. 1. PRISMA flow chart showing the screening process. 


\section{Quality assessment (n 51)}

Two reviewers (M.A.B.K. and P.M.) independently performed a quality assessment of the fifty-one studies identified as eligible in the screening (online Supplementary Appendix 2). We applied a validated Newcastle-Ottawa Quality Assessment Scale to assess the quality of the studies that were included in the review ${ }^{(29-31)}$. Quality scores obtained via the Newcastle-Ottawa scale for cross-sectional, cohort studies and case-control studies were used to assess selection, comparison and outcomes. Score disagreements were resolved through a discussion between M.A.B.K. and P.M., and a final consensual rating was assigned to each study. Studies six or more stars were considered high quality and were included in the review. Studies with fewer than six stars were excluded (online Supplementary Appendix 2).

\section{Results ( $n$ 41)}

\section{Categorisation of determinants}

Ten studies met the inclusion criteria covering pandemic confinements and their effects. These were then further subdivided into the following five main categories:

a. Demographic determinants

b. The impact of pandemic confinements on body weight

c. Dietary changes and other lifestyle behaviour changes during the confinement

d. Behaviour changes observed in obese participants

e. Determinants of obesity during pandemic confinements.

Our search yielded 5070 records of which 2361 unique studies remained after deduplication. After applying the inclusion and exclusion criteria in the title and abstract screening, seventy-eight articles were eligible for full-text screening (Fig. 1). We excluded ten studies based on a quality assessment of the results, and twenty-seven studies were excluded based on reasons presented in the Preferred Reporting Items for Systematic Reviews and Meta-analyses flow diagram (Fig. 1). The range of observations covered dietary choices ${ }^{(13,20-25,32-35,35-46)}$, lifestyle changes in children ${ }^{(23,35,47-50)}$, physical activity levels ${ }^{(32-34,36,37-40,42,45,46,48,51,52-59)}$, psychosocial factors ${ }^{(21,22,25,37,43}$, $44,50,51,54,55,57,60,61)$, socio-economic factors ${ }^{(22,36,47,51,53,60)}$ and sleeping patterns ${ }^{(25,45,50,62)}$.

\section{Demographic determinants (study and sample characteristics) (n 41)}

Table 2 describes the characteristics of each of the forty-one included studies. All of the studies were published in 2020 and 2021. Two studies were from preprints and were included after assessing their qualities individually ${ }^{(21,51)}$.

The included studies had the following countries of origin: Brazil $^{(58)}$, China ${ }^{(39,56)}$, Croatia ${ }^{(50,63)}$, France ${ }^{(46,51,55)}$, Jordan ${ }^{(57)}$,

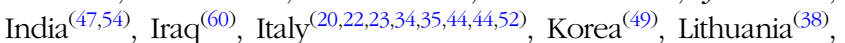
Netherlands $^{(59)}$, Poland ${ }^{(24,41,50)}$, Spain ${ }^{(43,45,62)}$, Turkey ${ }^{(42,48,64,65)}$, United Arab Emirates ${ }^{(32)}$, UK ${ }^{(53)}$ and the USA ${ }^{(25,37,40,66)}$ '. Furthermore, multi-regional studies conducted intercontinentally ${ }^{(21)}$, among eighteen countries in the Middle East and
North Africa region ${ }^{(33)}$, and Paraguay and Italian-based multinational researches ${ }^{(61)}$ are included in our analysis.

Altogether, the studies enrolled 469362 participants. The participants ranged in age from 6 to 86 years, and the mean ages for the individuals studied ranged from 9.9 to 74.3 years. The proportion of female participants ranged from $37 \%$ to $100 \%$. The number of participants in the included studies ranged from 41 to 381 564. All studies included both male and female participants except one study ${ }^{(36)}$. The duration of confinement for the selected studies for this systematic review ranged between 1 and 24 weeks.

\section{Impact of confinement on body weight}

In our study, $7 \cdot 2-72 \cdot 4 \%$ of all participants including both adults and children experienced an increase in body weight during the confinement periods ${ }^{(20,22,24,25,32,34-36,38-40,42-51,54,56-61,63-}$ ${ }^{67)}$ (Fig. 2). The mean weight gain ranged from $0 \cdot 6$ (SD 1.3) to $3 \cdot 0$ (SD 2.4) kg. There was a higher weight gain among participants who self-reported stress ${ }^{(25,44,54,55,57,60,61)}$, anxiety and depression $^{(22,51,57,60,61)}$. Weight loss was observed in $11 \cdot 1-$ $32 \cdot 0 \%$ of participants $(20,24,32,34,36,39,50,51,54,59,64,67)$. The mean experienced weight loss ranged from 2.0 (sD 1.4) to 2.9 (sD 1.5$) \mathrm{kg}$.

\section{Dietary and other lifestyle behaviour changes during confinement}

Table 3 describes dietary and behavioural changes that were caused by pandemic-related confinements. Most studies reported an increase in food intake associated with increased snacking $(20,22,24,25,32,33,35,36,38,40,42,43,50,51,54,67)$ and all these studies documenting perceived weight gain $^{(20,22,24,25,32,33,35,36,38,40,42,43,50,51,54,67)}$.

Appetite was modified either negatively or positively and was associated with employment change, suspension or working from home $e^{(20,51,52,57)}$ or due to suspension of school attendance ${ }^{(23,47-49)}$.

The initiating factors were as follows: response to smell and sight of food ${ }^{(24,25)}$, boredom, binge eating and food cravings $(24,25,40,42,44,54,66)$, snacking post dinner ${ }^{(25,32,33,38)}$ and visual stimulation through social media ${ }^{(32)}$. A significant correlation was observed between snacking, the consumption of high density processed food and a higher $\mathrm{BMI}^{(20,22,24,38)}$. Increased energy intake by 10-49.4\% was observed among study participants ${ }^{(20,22,24,25,32-38,40,42-44,51,54,66)}$, particularly those with an increased consumption of high density processed foods ${ }^{(20,22,23,34-36,38,40,42,43,50,67)}$, female $\operatorname{sex}^{(20,34,38,42,51,63)}$ or with a higher BMI ${ }^{(20,22-24,36,38,43,44,48,63)}$. There was an increase in the number of meals eaten per day ${ }^{(23,35,36,44,67)}$ and participants ate more than usual ${ }^{(34,38,42,54)}$. The proportion of respondents engaged in cooking increased from $40 \%$ to $62 \%$ in our study sample $(24,32,38,51)$. Likewise, consumption of homemade recipes increased ${ }^{(22,23,32,33,38,51)}$ and eating homemade desserts increased compared with prelockdown ${ }^{(20,22,23,32,34,38,67)}$

Less than one-third of the surveyed participants consumed fresh fruits and vegetables on a daily basis, while a similar number consumed sweets and desserts every day ${ }^{(20,21,24,32,36,38,39,51)}$. 
Table 2. Characteristics of included studies

(Mean values and standard deviations)

\begin{tabular}{|c|c|c|c|c|c|c|c|c|c|c|c|c|c|c|}
\hline S. no & $\begin{array}{l}\text { First author, year, } \\
\text { country }\end{array}$ & $\begin{array}{l}\text { Number of } \\
\text { participants }\end{array}$ & Study design & Instrument used & Local setting/target population & $\begin{array}{l}\text { Survey ques- } \\
\text { tions type }\end{array}$ & $\begin{array}{l}\text { Proportion of } \\
\text { female partic- } \\
\text { ipants (\%) }\end{array}$ & $\begin{array}{l}\text { Age of par- } \\
\text { ticipants } \\
\text { range } \\
\text { (years) }\end{array}$ & $\begin{array}{l}\text { Mean age of partici- } \\
\text { pants (SD) }\end{array}$ & & $\begin{array}{c}\text { Mean BMI/Centile } \\
\text { of participants }\end{array}$ & & $\begin{array}{l}\text { Mean weight } \\
\text { (kg) of partici- } \\
\text { pants }\end{array}$ & \\
\hline 1 & $\begin{array}{l}\text { Adibelli, et al., 2020, } \\
\text { Turkey }{ }^{(48)} \text {. }\end{array}$ & 597 & $\begin{array}{l}\text { Cross-sectional } \\
\text { study }\end{array}$ & Online survey & $\begin{array}{l}\text { Children aged } 7-13 \text { years and } \\
\text { their parents }\end{array}$ & Validated & 55.8 & $\begin{array}{l}7-13 \text { (child) } \\
26-57 \\
\quad \text { (parent) }\end{array}$ & $\begin{array}{l}9.87 \pm 1.99 \text { (chil- } \\
\text { dren) } \\
37.63 \pm 5.83 \\
\text { (parents) }\end{array}$ & & $\mathrm{NR}$ & & NR & \\
\hline 2 & $\begin{array}{l}\text { Ahmed, et al., 2020, } \\
\text { Iraq( }^{(0)} \text {. }\end{array}$ & 765 & $\begin{array}{l}\text { Prospective } \\
\text { cross-sec- } \\
\text { tional case } \\
\text { series study }\end{array}$ & $\begin{array}{l}\text { Face-to-face inter- } \\
\text { view }\end{array}$ & Patients visiting bariatric clinic & Validated & 39.4 & $<20->70$ & $\mathrm{NR}$ & & NR & & 73 & \\
\hline 3 & $\begin{array}{l}\text { Athanasiadis, et al., } \\
2020, \text { USA }^{(40)}\end{array}$ & 208 & Cross-sectional & Online survey & Postoperative bariatric patients & Validated & 86 & NR & 48.9 & 11.2 & NR & & 92.1 & 23.6 \\
\hline 4 & $\begin{array}{l}\text { Błaszczyk-Bebenek, } \\
\text { et al., 2020, } \\
\text { Poland (41). }\end{array}$ & 312 & $\begin{array}{l}\text { Observational } \\
\text { retrospective }\end{array}$ & $\begin{array}{l}\text { Self-administered } \\
\text { web-based } \\
\text { questionnaire }\end{array}$ & Healthy adults & Validated & 64.1 & NR & $41 \cdot 12$ & 13.05 & 24.98 & 4.33 & 73.47 & 16.65 \\
\hline 5 & $\begin{array}{l}\text { Chagué et al., 2020, } \\
\text { France }^{(46)} \text {. }\end{array}$ & 124 & $\begin{array}{l}\text { Cross-sectional } \\
\text { survey }\end{array}$ & Phone interviews & Congestive heart failure patients & New & 39.5 & NR & 71.0 & 14.0 & 28.2 & 5.4 & & \\
\hline 6 & $\begin{array}{l}\text { Chopra, et al., 2020, } \\
\text { India }{ }^{(54)} \text {. }\end{array}$ & 995 & $\begin{array}{l}\text { Cross-sectional } \\
\text { study }\end{array}$ & Online survey & Adults $\geq 18$ years & Validated & 41.5 & $\leq 30>30$ & 33.33 & 14.5 & $24.8 \pm 4.7 \mathrm{~kg} / \mathrm{m}^{2}$ & & NR & \\
\hline 7 & $\begin{array}{l}\text { Cransac-Miet, et al., } \\
\text { 2021, France }{ }^{(55)} \text {. }\end{array}$ & 195 & $\begin{array}{l}\text { Cross-sectional } \\
\text { population- } \\
\text { based study }\end{array}$ & Phone interview & $\begin{array}{l}\text { Patients with chronic coronary } \\
\text { syndromes }\end{array}$ & New & 39 & NR & 65.5 & $11 \cdot 1$ & NR & & NR & \\
\hline 8 & $\begin{array}{l}\text { Deschasaux-Tanguy } \\
\text { et al,, 2020, } \\
\text { France(51). }\end{array}$ & 37252 & $\begin{array}{l}\text { Cross-sectional } \\
\text { survey }\end{array}$ & $\begin{array}{l}\text { Self-administered } \\
\text { web-based } \\
\text { questionnaire }\end{array}$ & NutriNet-Santé cohort & Validated & $52 \cdot 3$ & $18-80+$ & $52 \cdot 1$ & 16.6 & NR & & NR & \\
\hline 9 & $\begin{array}{l}\text { Di Santo, et al., 2020, } \\
\text { ltaly }{ }^{(34)} \text {. }\end{array}$ & 126 & $\begin{array}{l}\text { Cross-sectional } \\
\text { observational } \\
\text { study }\end{array}$ & $\begin{array}{l}\text { Telephone inter- } \\
\text { view }\end{array}$ & Mild cognitive impairment patients & Validated & 81 & $60-87$ & 74.29 & 6.51 & NR & & NR & \\
\hline 10 & $\begin{array}{l}\text { Di Renzo et al., 2020, } \\
\text { Italy }{ }^{(20)} \text {. }\end{array}$ & 3533 & $\begin{array}{l}\text { Cross-sectional } \\
\text { survey }\end{array}$ & $\begin{array}{l}\text { Self-administered } \\
\text { web-based } \\
\text { questionnaire }\end{array}$ & General public & Validated & $75 \cdot 1$ & $12-86$ & \pm 13.53 & & 27.66 & $4 \cdot 10$ & 66.87 & $13 \cdot 16$ \\
\hline 11 & $\begin{array}{l}\text { Dogaš, et al., 2021, } \\
\text { Croatiai(63). }\end{array}$ & 3027 & $\begin{array}{l}\text { Cross-sectional } \\
\text { study }\end{array}$ & $\begin{array}{l}\text { Online question- } \\
\text { naire }\end{array}$ & General public & Validated & 70.1 & NR & 40 & $\begin{array}{r}30- \\
50\end{array}$ & 74.03 & 16.03 & 24.64 & 4.22 \\
\hline 12 & $\begin{array}{l}\text { Dondi, et al., 2021, } \\
\text { Italy }\end{array}$ & 5811 & $\begin{array}{l}\text { Cross-sectional } \\
\text { study }\end{array}$ & Online survey & $\begin{array}{l}\text { Italian resident parents of chil- } \\
\text { dren } \leq 18 \text { years }\end{array}$ & Validated & 91.7 & $\leq 30->50$ & NR & & NR & & NR & \\
\hline 13 & $\begin{array}{l}\text { Dragun, et al., 2020, } \\
\text { Split, Croatia( }{ }^{(50)} \text {. }\end{array}$ & 531 & $\begin{array}{l}\text { Cross-sectional } \\
\text { study }\end{array}$ & Online survey & Students & Validated & 63.8 & $\begin{array}{l}17-24 \\
\text { (median) }\end{array}$ & 18.0 & 6.0 & 21.4 & 3.3 & NR & \\
\hline 14 & $\begin{array}{l}\text { Drywień, et al., 2020, } \\
\text { Poland }{ }^{(36)} \text {. }\end{array}$ & 1769 & $\begin{array}{l}\text { Cross-sectional } \\
\text { study }\end{array}$ & Online survey & Polish women & Validated & 100 & $\geq 18$ & NR & & NR & & NR & \\
\hline 15 & $\begin{array}{l}\text { Dihogo Gama de } \\
\text { Matos, et al., 2020, } \\
\text { Brazi|(158). }\end{array}$ & 426 & $\begin{array}{l}\text { Cross-sectional } \\
\text { study }\end{array}$ & $\begin{array}{l}\text { Self-administered } \\
\text { web-based } \\
\text { questionnaire }\end{array}$ & General public & Validated & 49.1 & $7-80$ & $\begin{array}{l}\text { Multiple range from } \\
\text { children to el- } \\
\text { derly }\end{array}$ & & $\begin{array}{l}\text { Multiple stratified per } \\
\text { age }\end{array}$ & & $\begin{array}{l}\text { Multiple weight } \\
\text { stratified per } \\
\text { age }\end{array}$ & \\
\hline 16 & $\begin{array}{l}\text { Gentile, et al., 2020, } \\
\text { Vasto- Italy and } \\
\text { Paraguay(61). }\end{array}$ & 110 & $\begin{array}{l}\text { Observational } \\
\text { study }\end{array}$ & $\begin{array}{l}\text { Phone-based clini- } \\
\text { cal follow-up } \\
\text { and survey }\end{array}$ & $\begin{array}{l}\text { Psychiatric } \\
\text { outpatients }\end{array}$ & Validated & 54.5 & NR & 38.6 & 14.1 & NR & & $\mathrm{NR}$ & \\
\hline 17 & $\begin{array}{l}\text { Giustino et al., 2020, } \\
\text { Italy }\end{array}$ & 802 & $\begin{array}{l}\text { Cross-sectional } \\
\text { study }\end{array}$ & $\begin{array}{l}\text { Self-administered } \\
\text { web-based } \\
\text { questionnaire }\end{array}$ & Physically active participants & Validated & 51 & NR & $32 \cdot 27$ & 12.81 & 23.44 & 3.33 & $67 \cdot 13$ & 13.41 \\
\hline 18 & $\begin{array}{l}\text { He, et al., 2020, } \\
\text { China }{ }^{(39)} \text {. }\end{array}$ & 339 & $\begin{array}{l}\text { Cross-sectional } \\
\text { study }\end{array}$ & Online survey & Adults $\geq 18$ years & New & $52 \cdot 3$ & NR & $\begin{array}{l}\text { Males:36.4 (11.9) } \\
\text { Females: } 37.6 \\
\text { (12.4) }\end{array}$ & & NR & & $\begin{array}{l}\text { Female: } \\
51 \cdot 1 \pm 4 \cdot 1 \\
\text { Male: } 65.6 \pm 5.8\end{array}$ & \\
\hline
\end{tabular}


NS British Journal of Nutrition

Table 2. (Continued)

\begin{tabular}{|c|c|c|c|c|c|c|c|c|c|c|c|c|c|c|}
\hline S. no & $\begin{array}{l}\text { First author, year, } \\
\text { country }\end{array}$ & $\begin{array}{l}\text { Number of } \\
\text { participants }\end{array}$ & Study design & Instrument used & Local setting/target population & $\begin{array}{l}\text { Survey ques- } \\
\text { tions type }\end{array}$ & $\begin{array}{l}\text { Proportion of } \\
\text { female partic- } \\
\text { ipants (\%) }\end{array}$ & $\begin{array}{l}\text { Age of par- } \\
\text { ticipants } \\
\text { range } \\
\text { (years) }\end{array}$ & $\begin{array}{l}\text { Mean age of partici- } \\
\text { pants (SD) }\end{array}$ & & $\begin{array}{l}\text { Mean BMI/Centile } \\
\text { of participants }\end{array}$ & & $\begin{array}{l}\text { Mean weight } \\
\text { (kg) of partici- } \\
\text { pants }\end{array}$ & \\
\hline 19 & $\begin{array}{l}\text { Ismail, et al., 2020, } \\
\text { MENA Region }{ }^{(3)} .\end{array}$ & 2970 & Cross-sectional & $\begin{array}{l}\text { Online question- } \\
\text { naire }\end{array}$ & Adults $\geq 18$ years & Validated & $71 \cdot 6$ & $18->55$ & NR & & NR & & NR & \\
\hline 20 & $\begin{array}{l}\text { Ismail, et al., 2020, } \\
\text { UAE }{ }^{(33)} \text {. }\end{array}$ & 1012 & $\begin{array}{l}\text { Cross-sectional } \\
\text { study }\end{array}$ & Online survey & Adults $\geq 18$ years & Validated & 75.9 & $18-\geq 36$ & NR & & NR & & NR & \\
\hline 21 & $\begin{array}{l}\text { Jia, et al., 2020, } \\
\text { China } \\
(56) \text {. }\end{array}$ & 10082 & $\begin{array}{l}\text { Retrospective } \\
\text { study }\end{array}$ & $\begin{array}{l}\text { online question- } \\
\text { naire and }\end{array}$ & Chinese youth & Validated & 72 & $16-28$ & 19.8 & $2 \cdot 3$ & $21.8 \mathrm{~kg} / \mathrm{m}^{2}$ & & NR & \\
\hline 22 & $\begin{array}{l}\text { Jimenez, et al., 2021, } \\
\text { Spain }{ }^{(43)} \text {. }\end{array}$ & 603 & $\begin{array}{l}\text { Cross-sectional } \\
\text { study }\end{array}$ & Online survey & Patients attending obesity clinic & New & 27.5 & $18-\geq 55$ & NR & & 34.2 & 7.0 & NR & \\
\hline 23 & $\begin{array}{l}\text { Kang, et al., 2021, } \\
\text { Korea }^{(49)} \text {. }\end{array}$ & 226 & $\begin{array}{l}\text { Retrospective } \\
\text { cohort study }\end{array}$ & $\begin{array}{l}\text { Retrospective } \\
\text { review of medi- } \\
\text { cal records }\end{array}$ & $\begin{array}{l}\text { Children followed-up at the } \\
\text { growth clinic }\end{array}$ & $\begin{array}{l}\text { Not applicable } \\
\text { (anthropometric } \\
\text { and labora- } \\
\text { tory parame- } \\
\text { ters) }\end{array}$ & 57.5 & $4-18$ & $10.5(8.7-11.4)$ IQR & & $\begin{array}{l}0.2(1.3) \text { anthropo- } \\
\text { metric } z \text { scores }\end{array}$ & & $\begin{array}{l}0.1(1.2) \\
\text { anthropomet- } \\
\text { ric } z \text { scores }\end{array}$ & \\
\hline 24 & $\begin{array}{l}\text { Karatas, et al., 2020, } \\
\text { Istanbu|(65). }\end{array}$ & 140 & $\begin{array}{l}\text { Prospective } \\
\text { observational } \\
\text { case-control } \\
\text { study }\end{array}$ & $\begin{array}{l}\text { Physical and bio- } \\
\text { chemical } \\
\text { parameters }\end{array}$ & $\begin{array}{l}\text { Known confirmed type } 2 \text { diabetes } \\
\text { patients matched with healthy } \\
\text { patients in outpatient clinic }\end{array}$ & None & $\begin{array}{l}\text { Non-diabetic: } \\
56 \cdot 4 \\
\text { Diabetic: } \\
68 \cdot 2\end{array}$ & NR & $\begin{array}{l}\text { Non-diabetic: } \\
52 \cdot 61 \pm 4 \cdot 88 \\
\text { Diabetic: } \\
54.81 \pm 10.53\end{array}$ & & $\begin{array}{l}\text { Total mean } \\
31.63 \pm 3.57 \mathrm{~kg} / \mathrm{m}^{2} \\
\text { Non-diabetic: } \\
31.63 \pm 3.57 \\
\text { Diabetic } 33.44 \pm 6.48\end{array}$ & & $\begin{array}{l}\text { Non-diabetic: } \\
85.56 \pm 10.53 \\
\text { Diabetic: } \\
87.83 \pm 18.27\end{array}$ & \\
\hline 25 & $\begin{array}{l}\text { Keel PhD, et al., 2020, } \\
\text { USA }{ }^{(37)} \text {. }\end{array}$ & 90 & $\begin{array}{l}\text { Prospective } \\
\text { study }\end{array}$ & Online surveys & $\begin{array}{l}\text { Undergraduate psychology stu- } \\
\text { dents }\end{array}$ & Validated & 88 & NR & $19.45(1.26)$ years & & 22.93 & & 63.87 & \\
\hline 26 & $\begin{array}{l}\text { Kriaucioniene, et al., } \\
\text { 2020, Lithuania }{ }^{(38)} \text {. }\end{array}$ & 2447 & $\begin{array}{l}\text { Cross-sectional } \\
\text { study }\end{array}$ & $\begin{array}{l}\text { Self-administered } \\
\text { web-based } \\
\text { questionnaires }\end{array}$ & General public & Validated & 87.8 & $>18-\geq 51$ & NR & & NR & & NR & \\
\hline 27 & $\begin{array}{l}\text { Malkawi, et al., 2020, } \\
\text { Jordan }{ }^{(57)} \text {. }\end{array}$ & 2103 & $\begin{array}{l}\text { Cross-Sectional } \\
\text { Study }\end{array}$ & Online survey & $\begin{array}{l}\text { Mothers living in Jordan who } \\
\text { have at least one child } \\
\text { between the ages of } 4-18 \\
\text { years }\end{array}$ & Validated & NR & $\begin{array}{l}\text { Mother's } \\
\text { age } \\
\text { range: } \\
20-60 \\
\text { years }\end{array}$ & 36.2 years & & NR & & NR & \\
\hline 28 & $\begin{array}{l}\text { Marchitelli, et al., } \\
\text { 2020, Italy }\end{array}$ & 110 & Cross-sectional & Online survey & $\begin{array}{l}\text { Day care patients in hospitals for } \\
\text { obesity management }\end{array}$ & Validated & 71 & NR & $\begin{array}{l}\text { No psychiatric ill- } \\
\text { ness: } 18-75 \\
\text { years } \\
\text { ( } M=47 \cdot 24 \\
\text { SD }=14 \cdot 3 \text { ) } \\
\text { Psychiatric illness: } \\
\text { 18-74 years } \\
(\mathrm{M}=46 \cdot 38 \\
\text { SD }=14 \cdot 5)\end{array}$ & & $\begin{array}{l}\text { No psychiatric illness: } \\
40 \cdot 19 \mathrm{~kg} / \mathrm{m}^{2} \\
(\mathrm{sD}=6 \cdot 8, \text { range: } \\
27-60) \\
\text { Psychiatric illness: } \\
39 \cdot 88 \mathrm{~kg} / \mathrm{m}^{2} \\
(\mathrm{sD}=6 \cdot 8, \text { range: } \\
28-55)\end{array}$ & & NR & \\
\hline 29 & $\begin{array}{l}\text { Martínez-de-Quel } \\
\text { et al., 2021, } \\
\text { Spain (45). }\end{array}$ & 161 & $\begin{array}{l}\text { Longitudinal } \\
\text { observational } \\
\text { study }\end{array}$ & Online survey & Spanish adults & Validated & 37 & $19-65$ & $35 \cdot 0$ & $11 \cdot 2$ & 23.7 & 4 & $67 \cdot 3$ & 14.8 \\
\hline 30 & $\begin{array}{l}\text { Mason, et al., 2021, } \\
\text { USA }\end{array}$ & 1820 & $\begin{array}{l}\text { Longitudinal pro- } \\
\text { spective } \\
\text { cohort study }\end{array}$ & Online survey & High schools & Validated & 61 & NR & 19.28 & & NR & & $70.3 \mathrm{~kg}$ & \\
\hline 31 & $\begin{array}{l}\text { Mitchell et al., 2020, } \\
\text { USA }\end{array}$ & 3 81564 & $\begin{array}{l}\text { Observational, } \\
\text { retrospective, } \\
\text { cohort study }\end{array}$ & $\begin{array}{l}\text { Noom app - } \\
\text { mobile behav- } \\
\text { iour change } \\
\text { weight loss pro- } \\
\text { gramme }\end{array}$ & $\begin{array}{l}\text { App-based food data from a digi- } \\
\text { tal behaviour change } \\
\text { weight loss programme }\end{array}$ & $\begin{array}{l}\text { App-based vali- } \\
\text { dated }\end{array}$ & 83.4 & $\geq 18$ & 47.76 & 13.59 & NR & & 85.57 & 20.4 \\
\hline
\end{tabular}


NS British Journal of Nutrition

Table 2. (Continued)

\begin{tabular}{|c|c|c|c|c|c|c|c|c|c|c|c|c|c|c|}
\hline S. no & $\begin{array}{l}\text { First author, year, } \\
\text { country }\end{array}$ & $\begin{array}{l}\text { Number of } \\
\text { participants }\end{array}$ & Study design & Instrument used & Local setting/target population & $\begin{array}{l}\text { Survey ques- } \\
\text { tions type }\end{array}$ & $\begin{array}{l}\text { Proportion of } \\
\text { female partic- } \\
\text { ipants (\%) }\end{array}$ & $\begin{array}{l}\text { Age of par- } \\
\text { ticipants } \\
\text { range } \\
\text { (years) }\end{array}$ & $\begin{array}{l}\text { Mean age of partici- } \\
\text { pants (SD) }\end{array}$ & & $\begin{array}{l}\text { Mean BMI/Centile } \\
\text { of participants }\end{array}$ & & $\begin{array}{l}\text { Mean weight } \\
\text { (kg) of partici- } \\
\text { pants }\end{array}$ & \\
\hline 32 & $\begin{array}{l}\text { Önmez, et al., 2020, } \\
\text { Turkey }\end{array}$ & 101 & $\begin{array}{l}\text { Retrospective } \\
\text { observational } \\
\text { study }\end{array}$ & Questionnaire & $\begin{array}{l}\text { Diabetic patients attending poly- } \\
\text { clinics }\end{array}$ & Validated & 53.5 & $18-80$ & 55 & 13 & $30 \cdot 3$ & 5.5 & $84 \cdot 7 \pm 16 \cdot 4 \mathrm{~kg}$ & \\
\hline 33 & $\begin{array}{l}\text { Özden, et al., 2021, } \\
\text { Turkey }\end{array}$ & 1011 & $\begin{array}{l}\text { Cross-sectional } \\
\text { study }\end{array}$ & Online survey & Nursing students & Validated & 60 & NR & $19.97 \pm 3.11$ years & & NR & & NR & \\
\hline 34 & $\begin{array}{l}\text { Pellegrini et al., 2020, } \\
\text { Italy }\end{array}$ & 150 & $\begin{array}{l}\text { Observational } \\
\text { retrospective } \\
\text { study }\end{array}$ & $\begin{array}{l}\text { Telephone inter- } \\
\text { views cross- } \\
\text { sectional sur- } \\
\text { vey }\end{array}$ & $\begin{array}{l}\text { Obese patients in weight loss pro- } \\
\text { gramme }\end{array}$ & Validated & $76 \cdot 3$ & $18-75$ & 47.9 & $16 \cdot 0$ & 36.6 & 4.5 & 92 & 17 \\
\hline 35 & $\begin{array}{l}\text { Pietrobelli et al., 2020, } \\
\text { Italy(23). }\end{array}$ & 41 & $\begin{array}{l}\text { Longitudinal } \\
\text { observational } \\
\text { study/ques- } \\
\text { tionnaire }\end{array}$ & $\begin{array}{l}\text { In-person inter- } \\
\text { view/telephone } \\
\text { interviews } \\
\text { (parents) }\end{array}$ & School children & New & 46.35. & $6-18$ & 13 & 3.1 & $\begin{array}{l}30 \cdot 2 \pm 4 \cdot 1 \text { a and } B M I \\
\% \text { Centile } 98 \cdot 2 \pm 1 \cdot 4\end{array}$ & & $77 \cdot 4$ & 21.9 \\
\hline 36 & $\begin{array}{l}\text { Rogers et al., 2020, } \\
\text { UK(53). }\end{array}$ & 5820 & $\begin{array}{l}\text { Cross-sectional } \\
\text { survey }\end{array}$ & $\begin{array}{l}\text { Self-administered } \\
\text { web-based } \\
\text { questionnaire }\end{array}$ & General public & Validated & 88 & $20-70+$ & NR & & NR & & NR & \\
\hline 37 & $\begin{array}{l}\text { Romero-Blanco et al., } \\
\text { 2020, Spain(62). }\end{array}$ & 207 & $\begin{array}{l}\text { Longitudinal } \\
\text { observational } \\
\text { study }\end{array}$ & $\begin{array}{l}\text { Self-administered } \\
\text { questionnaire }\end{array}$ & Nursing students & Validated & $81 \cdot 6$ & $17-53$ & $20 \cdot 6$ & 4.62 & NR & & NR & \\
\hline 38 & $\begin{array}{l}\text { Ruissen, et al., 2021, } \\
\text { Netherlands }{ }^{(59)} \text {. }\end{array}$ & 435 & $\begin{array}{l}\text { Observational } \\
\text { cohort study }\end{array}$ & $\begin{array}{l}\text { Online question- } \\
\text { naire }\end{array}$ & $\begin{array}{l}\text { Type } 1 \text { and Type } 2 \text { diabetic } \\
\text { patients }\end{array}$ & Validated & 42 & $\geq 18$ & $\begin{array}{l}\text { Type 1 DM: } 50.1 \\
\text { ( } \pm 14 \cdot 9) \\
\text { Type 2 DM: } \\
62.5( \pm 11 \cdot 6)\end{array}$ & & $\begin{array}{l}\text { Type } 1 \text { DM: } 25 \cdot 9 \\
\quad( \pm 4 \cdot 3) \\
\text { Type } 2 \text { DM: } \\
30 \cdot 2( \pm 6 \cdot 1)\end{array}$ & & NR & \\
\hline 39 & $\begin{array}{l}\text { Shah, et al., 2020, } \\
\text { India(47). }\end{array}$ & 77 & $\begin{array}{l}\text { Observational } \\
\text { study }\end{array}$ & $\begin{array}{l}\text { Follow-up in out- } \\
\text { patient clinic }\end{array}$ & Children with type 1 diabetes & Validated & 58.4 & $5-20$ & $14 \pm 4$ years & & NR & & NR & \\
\hline 40 & $\begin{array}{l}\text { Sidor et al., 2020, } \\
\text { Poland } \\
\text { (24). }\end{array}$ & 1097 & $\begin{array}{l}\text { Cross-sectional } \\
\text { survey }\end{array}$ & $\begin{array}{l}\text { Self-administered } \\
\text { web-based } \\
\text { questionnaire }\end{array}$ & General public & New & $95 \cdot 1$ & $18-71$ & $27 \cdot 7$ & $9 \cdot 0$ & 21.523 .5 & 4.8 & 66.0 & 14.5 \\
\hline 41 & $\begin{array}{l}\text { Zachary et al., 2020, } \\
\text { USA }\end{array}$ & 173 & $\begin{array}{l}\text { Cross-sectional } \\
\text { survey }\end{array}$ & $\begin{array}{l}\text { Self-administered } \\
\text { web-based } \\
\text { questionnaire }\end{array}$ & General public & Validated & $55 \cdot 5$ & $\geq 18$ & 28.1 & 12.5 & $27 \cdot 0$ & 7.6 & NR & \\
\hline
\end{tabular}




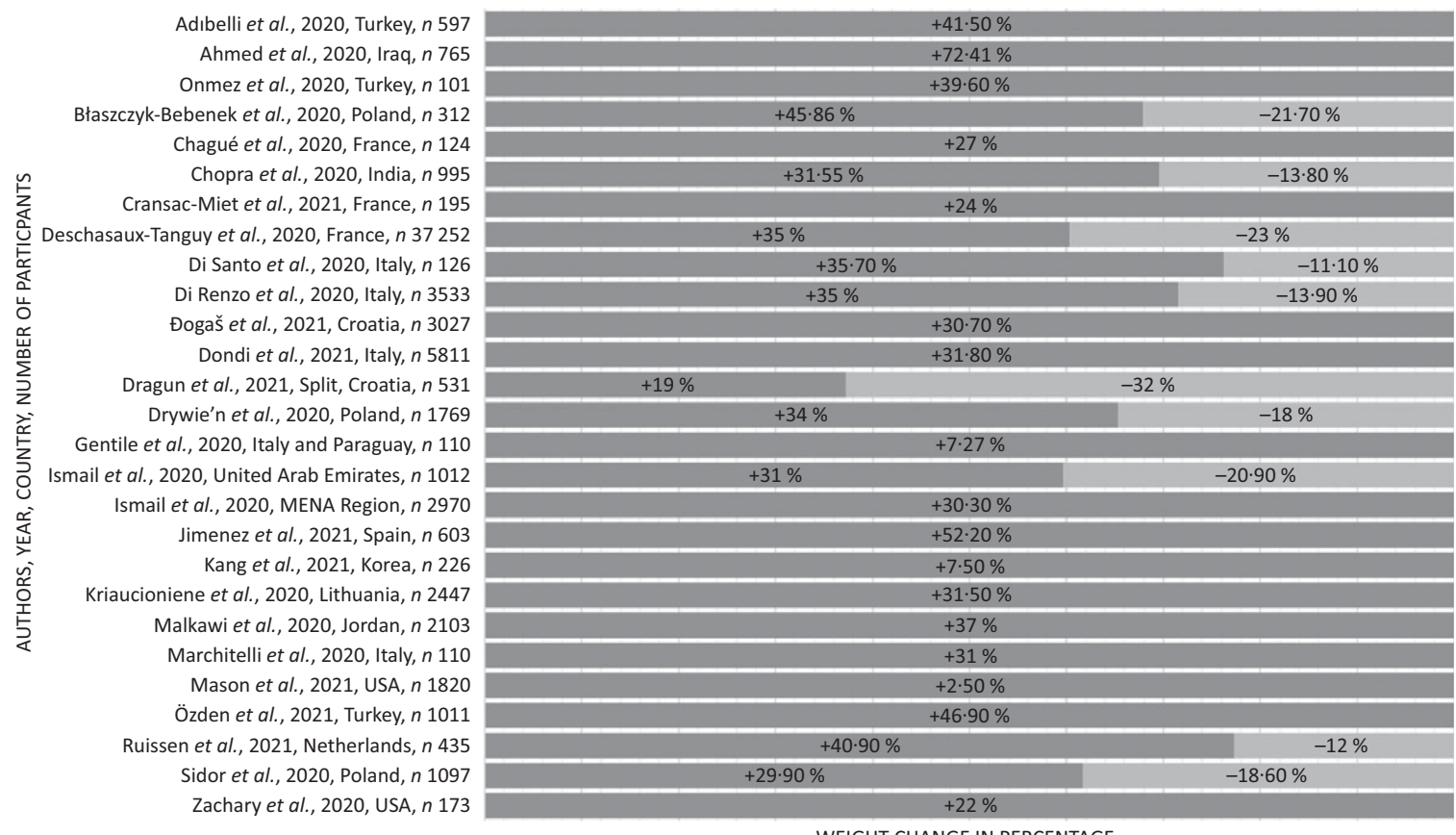

WEIGHT CHANGE IN PERCENTAGE

Fig. 2. Body weight changes during pandemic confinements. Selected studies showing percentage of body weight changes. For the full list of weight changes, please refer to Table 3. +, increase in weight; -, decrease in weight.

In contrast, some studies have shown a decrease in unhealthy

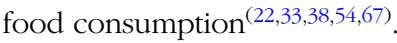

Where Mediterranean diet was followed, 18- to 30-year-olds were more compliant than other age groups ${ }^{(20)}$. Inverse associations were found between adherence to Mediterranean diet and $\mathrm{BMI}^{(20,50)}$. A total of $54 \%$ of respondents used leftovers for at least a third of meals, and those who shopped at farmers' markets or local or organic markets ate up leftovers more $(\mathrm{OR}=1.468$, $P<0.001)^{(20)}$. Among app users, mobile behavioural change app interaction was reduced by $9 \%{ }^{(21)}$. Eating in response to stress was associated with weight gain $25,34,58,66)$. There was increased alcohol consumption ${ }^{(24,46,55,61,67)}$ during the lockdown, while a decrease in alcohol consumption was also noted compared with pre-COVID-19 in another study $(20,46)$. There was an increase in cigarette smoking generally ${ }^{(46,50,55,63)}$ while in contrast, $3.3 \%$ of the smokers surveyed reported reduced smoking during quarantine ${ }^{(19)}$.

Although the participants reported spending more time in bed before lockdown ${ }^{(23,25,54,56)}$, the overall sleep quality was worse $^{(45,54,62)}$. In contrast, secondary school students felt refreshed on awakening and increased sleeping hours ${ }^{(50)}$. Weight gain was reported by others to be related to decreased night-time sleep and reduced physical activity time ${ }^{(25,40,59)}$

Sedentary lifestyle and screen time increased during the lockdown ${ }^{(23,25,37,46,54-56)}$. Those participants who were not currently working or those who started working from home felt that they gained more weight compared with participants who did not have a change in job routine ${ }^{(20,20,51,57)}$

Physical activity altered by varying amounts, reduced in some studies to between 18 and $84 \%^{(22,23,33,34,39,46,51,53,54,58)}$. People who were already overweight or obese engaged in less physical activity and had decreased energy expenditure during lockdown ${ }^{(36,38,43,51-53,55,58)}$. Obese children spent less time participating in sports activities ${ }^{(23)}$

By contrast, studies reporting an increase in physical activity $^{(20,51)}$ found greater engagement in yoga/pilates, functional training, home training, and treadmill use and overall increased training frequency ${ }^{(20)}$.

\section{Behaviour changes observed in obese participants}

Weight gain was more common in those already overweight or obese prior to lockdown and in individuals with pre-existing difficulty in weight management ${ }^{(20,22-24,36,38,43,45,48,63)}$.

Increased snacking and food consumption were observed in participants with a higher $\mathrm{BMI}^{(23,24,32,33,67)}$. Many of the participants agreed that they consumed less fruits and vegetables on a daily basis ${ }^{(21,24,33,38,40,51)}$ but more high energy processed foods ${ }^{(22-24,40,43)}$

This intake was associated with an enhanced appetite and after-dinner hunger ${ }^{(20,36,38,44)}$. Obese children reported an increase in the number of meals eaten along with an increased consumption of sweetened drinks, potato chips and red meat ${ }^{(23)}$. A decrease in intensive physical activity was associated with obesity ${ }^{(53)}$. An inverse relationship was found between changes occurring in sporting activities and the number of meals consumed per day $(23,40,52,67)$. The participants self-reporting anxiety and depression displayed an estimated weight $\operatorname{gain}^{(22,44,54,55,57,61)}$

\section{Determinants that can influence body weight during pandemics}

Table 4 describes the determinants of body weight changes during the pandemic. Many determinants that can influence increased weight gain during confinement were identified via 


\section{NS British Journal of Nutrition}

Table 3. Behavioural and dietary changes related to pandemic confinements

\begin{tabular}{|c|c|c|c|c|c|c|c|c|c|c|c|}
\hline $\begin{array}{l}\text { S. } \\
\text { no }\end{array}$ & $\begin{array}{l}\text { First author, } \\
\text { year, country }\end{array}$ & $\begin{array}{l}\text { Duration of } \\
\text { confinement } \\
\text { during which } \\
\text { study was } \\
\text { conducted } \\
\text { (weeks) }\end{array}$ & Outcome area of focus & $\begin{array}{l}\text { Number of } \\
\text { participants }\end{array}$ & $\begin{array}{l}\text { Weight gain of the } \\
\text { participant }(\%) \\
\text { (weight gain in } \mathrm{kg} \text { ), } \\
\text { weight loss of the } \\
\text { participant }(\%) \\
\text { (weight loss in } \mathrm{kg} \text { ) }\end{array}$ & $\begin{array}{l}\text { Energy intake/ } \\
\text { food intake }\end{array}$ & Snacking & $\begin{array}{l}\text { Fresh product } \\
\text { (fruits and vegeta- } \\
\text { ble) }\end{array}$ & Physical activity & Alcohol & $\begin{array}{l}\text { Dietary patterns and other behaviour } \\
\text { changes identified during the confinement }\end{array}$ \\
\hline 1 & $\begin{array}{l}\text { Adibelli, et al., } \\
2020, \\
\text { Turkey(48). }\end{array}$ & 4 weeks & $\begin{array}{l}\text { Health related quality } \\
\text { of life }\end{array}$ & 597 & $\uparrow 41.5 \%$ & NR & NR & NR & NR & NR & $\begin{array}{l}\text { Quality of life score mean } 73.91 \pm 8.44 \\
\text { Increase in sleep time of } 34.2 \% \\
\text { Increase Internet usage of } 69.3 \%\end{array}$ \\
\hline 2 & $\begin{array}{l}\text { Ahmed, et al., } \\
2020, \\
\operatorname{lraq}^{(60)}\end{array}$ & 1-9 weeks & Body weight & 765 & $\uparrow 72.41 \%$ & NR & NR & NR & NR & NR & $\begin{array}{l}\text { One-third of them became emotionally } \\
\text { unstable during the outbreak } \\
\text { Even after the isolation process } \\
\text { calmed down, the stress was present in } \\
\text { more patients compared with the } \\
\text { period of the outbreak }\end{array}$ \\
\hline 3 & $\begin{array}{l}\text { Athanasiadis, } \\
\text { et al., } 2020 \text {, } \\
\text { USA }^{(40)} \text {. }\end{array}$ & 5 & $\begin{array}{l}\text { Factors attributed to } \\
\text { weight gain }\end{array}$ & 208 & $\begin{array}{l}2+4 \cdot 2 \mathrm{~kg} \text { in } \\
\text { patients }>18 \\
\text { months post-bari- } \\
\text { atric surgery }\end{array}$ & Increased & $\uparrow 62.6 \%$ & $\downarrow 45.5 \%$ & $\downarrow 55.2 \%$ & $\uparrow 40.1 \%$ & $\begin{array}{l}19.5 \% \text { reported increase in binge eating } \\
48.2 \% \text { reported loss of control when eating } \\
\text { Weight gain of }>2 \mathrm{~kg} \text { in patients }>18 \text {-month } \\
\text { post-bariatric surgery }\end{array}$ \\
\hline 4 & $\begin{array}{l}\text { Btaszczyk- } \\
\text { Bebenek, } \\
\text { et al., 2020, } \\
\text { Poland(41). }\end{array}$ & $5-8$ & $\begin{array}{l}\text { Nutrition behaviour } \\
\text { changes during lock- } \\
\text { down }\end{array}$ & 312 & $\begin{array}{l}\uparrow 45.86 \%(0.56 \pm 2.43 \\
\mathrm{kg}) \\
\downarrow 21.72 \%\end{array}$ & $\begin{array}{l}\uparrow 11 \cdot 2 \% \text { in } \\
\text { number of } \\
\text { meals }\end{array}$ & $\begin{array}{l}\text { Trom } 72.8 \% \text { to } \\
\quad 77.9 \\
\quad(P<0.0001)\end{array}$ & $\begin{array}{l}\text { Pfrom } 63.8 \% \text { to } \\
\quad 64.7 \% \\
(P=0.7755)\end{array}$ & NR & Increased & $\begin{array}{l}\text { Increase of consumption of eggs, potatoes, } \\
\text { sweets and canned meat }\end{array}$ \\
\hline 5 & $\begin{array}{l}\text { Chagué et al., } \\
2020, \\
\text { France }^{(46)} \text {. }\end{array}$ & $6-7$ & $\begin{array}{l}\text { Impact of lockdown on } \\
\text { health indicators } \\
\text { and behaviours } \\
\text { among congestive } \\
\text { heart failure patients }\end{array}$ & 124 & $\uparrow 27.4 \%$ & NR & NR & NR & $\downarrow 41.9 \%$ & $\uparrow 4 \% \downarrow 15 \%$ & $\begin{array}{l}\text { Screen time increased by } 46 \% \text {. } \\
\text { Tobacco consumption increased in } 44.4 \% \\
\text { of current smokers. Adherence to strict } \\
\text { salt and fluid restriction decreased by } \\
14.5 \% \text {. Increase in heart failure symp- } \\
\text { toms in } 21.8 \%\end{array}$ \\
\hline 6 & $\begin{array}{l}\text { Chopra, et al., } \\
2020, \\
\text { India }{ }^{(54)} \text {. }\end{array}$ & $\begin{array}{l}\text { 20-22 } \\
\text { weeks }\end{array}$ & $\begin{array}{l}\text { Impact of COVID-19 on } \\
\text { lifestyle-related } \\
\text { behaviours: eating, } \\
\text { physical activity and } \\
\text { sleep behaviour }\end{array}$ & 995 & $\begin{array}{l}\uparrow 31.55 \% \\
\downarrow 13.87 \%\end{array}$ & Increased & Increased & $\uparrow 34 \vee 38 \%$ & $\downarrow 9.5 \%$ & Decreased & $\begin{array}{l}\text { In participants }<30 \text { years old, increase in } \\
\text { healthy food and restriction of unhealthy } \\
\text { meals } \\
\text { Increase stress amongst } \\
\text { participants }(25 \% \vee 38 \cdot 3 \%) \\
\text { significantly increased } \\
\text { Increase in daily sleeping hours, screen } \\
\text { time, sitting time at work, stress levels. } \\
\text { Decreased smoking }\end{array}$ \\
\hline 7 & $\begin{array}{l}\text { Cransac-Miet, } \\
\text { et al, } 2021, \\
\text { France }^{(55)}\end{array}$ & 4 & Lifestyle changes & 195 & $\uparrow 24 \%$ & NR & NR & NR & $\downarrow 25 \%$ & $\begin{array}{l}5 \% \text { increase in alco- } \\
\text { hol consumption }\end{array}$ & $\begin{array}{l}\text { Smoking increased by } 26 \% \\
\text { Screen time increased in } 65 \% \text { of patients }\end{array}$ \\
\hline 8 & $\begin{array}{l}\text { Deschasaux- } \\
\quad \text { Tanguy } \\
\text { et al., 2020, } \\
\text { France }^{(51)} \text {. }\end{array}$ & $2-6$ & $\begin{array}{l}\text { Changes in diet and } \\
\text { physical activity dur- } \\
\text { ing lockdown }\end{array}$ & 37252 & $\begin{array}{l}\uparrow 35 \%(1 \cdot 8 \pm 1 \cdot 3 \mathrm{~kg}) \\
\quad \downarrow 23 \%(2 \cdot 0 \pm 1 \cdot 4 \\
\mathrm{kg})\end{array}$ & $\uparrow 10 \% \downarrow 10 \%$ & $\uparrow 21.1 \%$ & $\downarrow 10.1 \%$ & $\begin{array}{l}\uparrow 52.8 \%, \\
\downarrow 18 \%\end{array}$ & $\uparrow 15 \%, \downarrow 12 \%$ & $\begin{array}{l}\text { Positive behavioural trends were observed } \\
\text { in those with higher educational attain- } \\
\text { ment with high income but negative } \\
\text { trends were reported when income was } \\
\text { lower } \\
\text { Positive behavioural trends were observed } \\
\text { in the overweight/obese population with } \\
\text { higher educational attainment who } \\
\text { expressed anxiety: reduced snacking: } \\
\text { reduced alcohol consumption: increased } \\
\text { more home cooking }\end{array}$ \\
\hline 9 & $\begin{array}{l}\text { Di Santo, } \\
\text { et al., 2020, } \\
\text { Italy }\left({ }^{(34)} \text {. }\right.\end{array}$ & $8-10$ & $\begin{array}{l}\text { Lifestyle, mental health } \\
\text { Weight change } \\
\text { Behavioural changes }\end{array}$ & 126 & $\uparrow 35.7 \%, \downarrow 11.1 \%$ & $\uparrow 19.2 \%$ & NR & NR & $\begin{array}{l}1 / 3 \text { of the subjects } \\
\text { decreased their } \\
\text { physical activity }\end{array}$ & $\begin{array}{l}\text { Decreased in drink- } \\
\text { ers } 12.4 \% \\
\text { Increase in alcoholic } \\
\text { drink intake } \\
\quad(44.4 \%) \\
\text { Two subjects started } \\
\text { drinking wine }\end{array}$ & $\begin{array}{l}1 / 6 \text { of participants decreased mental-stimu- } \\
\text { lating activities } \\
70 \% \text { reported an increase in idle time } \\
19.8 \% \text { were depressed, } 9.5 \% \text { anxious and } \\
9.5 \% \text { apathetic } \\
31.9 \% \text { consumed more sweets } \\
12.8 \% \text { ate preserved or frozen foods }\end{array}$ \\
\hline
\end{tabular}


NS British Journal of Nutrition

Table 3. (Continued)

\begin{tabular}{|c|c|c|c|c|c|c|c|c|c|c|c|}
\hline $\begin{array}{l}\text { S. } \\
\text { no }\end{array}$ & $\begin{array}{l}\text { First author, } \\
\text { year, country }\end{array}$ & $\begin{array}{l}\text { Duration of } \\
\text { confinement } \\
\text { during which } \\
\text { study was } \\
\text { conducted } \\
\text { (weeks) }\end{array}$ & Outcome area of focus & $\begin{array}{l}\text { Number of } \\
\text { participants }\end{array}$ & $\begin{array}{l}\text { Weight gain of the } \\
\text { participant }(\%) \\
\text { (weight gain in kg), } \\
\text { weight loss of the } \\
\text { participant }(\%) \\
\text { (weight loss in } \mathrm{kg} \text { ) }\end{array}$ & $\begin{array}{l}\text { Energy intake/ } \\
\text { food intake }\end{array}$ & Snacking & $\begin{array}{l}\text { Fresh product } \\
\text { (fruits and vegeta- } \\
\text { ble) }\end{array}$ & Physical activity & Alcohol & $\begin{array}{l}\text { Dietary patterns and other behaviour } \\
\text { changes identified during the confinement }\end{array}$ \\
\hline 10 & $\begin{array}{l}\text { Di Renzo } \\
\text { et al., 2020, } \\
\text { Italy } 200 \text {. }\end{array}$ & $2-4$ & $\begin{array}{l}\text { Lifestyle changes, eat- } \\
\text { ing habits, and } \\
\text { adherence to the } \\
\text { Mediterranean diet } \\
\text { during the COVID } \\
\text { lockdown }\end{array}$ & 3533 & $\uparrow 35 \%, \downarrow 13.9 \%$ & $\uparrow 34.4 \%$ & $\uparrow 25.6 \%$ & $\begin{array}{c}\uparrow 37.4 \%, \downarrow \\
35.8 \%\end{array}$ & $\uparrow 38.3 \%$ & NR & $\begin{array}{l}\text { Younger participants adhered better to the } \\
\text { Mediterranean diet } \\
\text { Overweight participants had poor adher- } \\
\text { ence to the Mediterranean diet } \\
9.1 \% \text { of participants slept more than } 9 \mathrm{~h} / \mathrm{d}\end{array}$ \\
\hline 11 & $\begin{array}{l}\text { Dogaš, et al., } \\
\text { 2021, } \\
\text { Croatia } \\
\end{array}$ & 2 & Lifestyle, mood & 3027 & $\uparrow 30.7 \%$ & NR & NR & NR & $\begin{array}{l}\text { Women decreased } \\
\text { their exercise } \\
\text { duration and fre- } \\
\text { quency from } \\
57.9 \pm 34.5 \text { to } \\
51 \cdot 1 \pm 37.7\end{array}$ & Increased & $\begin{array}{l}\text { Women smoked more cigarettes } \\
\begin{array}{l}(P<0.001) \\
\text { Increased frequency of feeling afraid } \\
(P<0.001) \text {, discouraged }(P<0.001) \text { and } \\
\text { feeling sad }(P<0.001) \text { in both sexes }\end{array}\end{array}$ \\
\hline 12 & $\begin{array}{l}\text { Dondi, et al., } \\
2021, \\
\text { Italy }{ }^{(35)} \text {. }\end{array}$ & 24 & $\begin{array}{l}\text { Perception of food } \\
\text { insecurity in children }\end{array}$ & 5811 & $\uparrow 31.8 \%$ & $\uparrow 27.3 \%$ & $\uparrow 60.3 \%$ & $\uparrow 14.0 \%$ & $\mathrm{NR}$ & NR & $\begin{array}{l}27.3 \% \text { Children were eating more food } \\
\text { there was an increase. } 60.3 \% \text { consump- } \\
\text { tion. } 14 \% \text { fruit juices } 10.4 \% \text { soft drinks. } \\
2.5 \% \text { reported inadequate food after the } \\
\text { pandemic }\end{array}$ \\
\hline 13 & $\begin{array}{l}\text { Dragun, et al., } \\
\text { 2020, Split, } \\
\text { Croatia(50). }^{\text {. }}\end{array}$ & 3-11 & $\begin{array}{l}\text { Lifestyle changes and } \\
\text { psychological state }\end{array}$ & 531 & $\begin{array}{l}\uparrow 19 \% \\
\downarrow 32 \%\end{array}$ & $\begin{array}{l}\text { No difference } \\
\text { in dietary } \\
\text { pattern }\end{array}$ & $\begin{array}{l}\text { Increased } \\
20-38 \%\end{array}$ & $\begin{array}{l}\text { Increased }(65.3 \% \\
\quad \text { v. } 58.6 \%)\end{array}$ & Unchanged & NR & $\begin{array}{l}\text { Improved sleep quality } 31.5 \% \text {. } \\
\text { Sleep hours increased } \\
\text { Increased intake of legumes ( } 60.6 \% v \text {. } \\
53.3 \%) \text {, fish }(32.8 \% \text { v. } 24.4 \%) \text { and } \\
\text { sweets ( } 30.5 \% \text { v. } 22.4 \%) \\
\text { Decreased intake of cereals }(24.1 \% v \text {. } \\
35.6 \%) \text {, nuts ( }(15.1 \% \text { v. } 18.9 \%) \text {, and } \\
\text { dairy products } \\
\text { Increase computer screen time due to on- } \\
\text { line learning }\end{array}$ \\
\hline 14 & $\begin{array}{l}\text { Drywień, et al., } \\
\text { 2020, } \\
\text { Poland( }{ }^{(36)} \text {. }\end{array}$ & $3-7$ & $\begin{array}{l}\text { Changes in body } \\
\text { weight due to } \\
\text { COVID-19 lockdown }\end{array}$ & 1769 & $\begin{array}{l}\uparrow 34 \% \\
\downarrow 18 \%\end{array}$ & $\begin{array}{l}\uparrow 65 \% \\
\downarrow 40 \%\end{array}$ & $\begin{array}{c}\text { TSalty snacks } \\
(30.4 \% \mathrm{v} \text {. } \\
11.3 \%)\end{array}$ & $\begin{array}{l}\text { } \text { Consumption of } \\
\text { vegetables } \\
(32.3 \% \mathrm{v} \text {. } \\
16.0 \%) \text {, fruit } \\
(23.8 \% \mathrm{v} \text {. } \\
14.3 \%) \text { in } \\
\text { those with } \\
\text { weight loss }\end{array}$ & $\begin{array}{l}\downarrow \text { In weight gainers } \\
\quad(60.7 \% \mathrm{v} \text {. } \\
31.6 \%)\end{array}$ & $\begin{array}{l}\uparrow \text { In alcohol who } \\
\text { gained weight } \\
(25.4 \% \text { v. } 4 \cdot 1 \%)\end{array}$ & $\begin{array}{l}\text { Unhealthy dietary changes. } \\
\text { Increase in screen time } \\
\text { In females, those who lost weight ate more } \\
\text { fruits, vegetable, pulses, seafood, } \\
\text { drank > } 500 \text { ml water and did not con- } \\
\text { sume alcohol } \\
\text { Females who gained weight had increased } \\
\text { consumption of sweetened spreads, } \\
\text { commercial pastry, confectionery, salty } \\
\text { snacks, fast food, sugar-sweetened bev- } \\
\text { erages, processed meat, ice-cream and } \\
\text { pudding and alcohol, decreased physical } \\
\text { activity }\end{array}$ \\
\hline 15 & $\begin{array}{l}\text { Dihogo Gama } \\
\text { de Matos, } \\
\text { et al., 2020, } \\
\text { Brazil( }{ }^{(58)} \text {. }\end{array}$ & 12 & $\begin{array}{l}\text { Effects of COVID-19 } \\
\text { social distancing on } \\
\text { physical activity, } \\
\text { stress levels, quality } \\
\text { of life }\end{array}$ & 426 & Increased & NR & NR & NR & $\downarrow 84 \%$ & NR & $\begin{array}{l}\text { The study shows an overall decrease in all } \\
\text { sections of quality of life as analysed by } \\
\text { the SF- } 36 \text {. } \\
\text { The elderly age group showed no signifi- } \\
\text { cant changes. } \\
\text { There has been increase in stress level } \\
\text { across adolescents, adults and elderly } \\
\text { age groups in both sexes }(P<0.05) \\
\text { although there is no difference of stress } \\
\text { levels across children }\end{array}$ \\
\hline
\end{tabular}


NS British Journal of Nutrition

Table 3. (Continued)

\begin{tabular}{|c|c|c|c|c|c|c|c|c|c|c|c|}
\hline $\begin{array}{l}\text { S. } \\
\text { no }\end{array}$ & $\begin{array}{l}\text { First author, } \\
\text { year, country }\end{array}$ & $\begin{array}{l}\text { Duration of } \\
\text { confinement } \\
\text { during which } \\
\text { study was } \\
\text { conducted } \\
\text { (weeks) }\end{array}$ & Outcome area of focus & $\begin{array}{l}\text { Number of } \\
\text { participants }\end{array}$ & $\begin{array}{l}\text { Weight gain of the } \\
\text { participant }(\%) \\
\text { (weight gain in } \mathrm{kg} \text { ), } \\
\text { weight loss of the } \\
\text { participant } \% \text { ) } \\
\text { (weight loss in } \mathrm{kg} \text { ) }\end{array}$ & $\begin{array}{l}\text { Energy intake/ } \\
\text { food intake }\end{array}$ & Snacking & $\begin{array}{l}\text { Fresh product } \\
\text { (fruits and vegeta- } \\
\text { ble) }\end{array}$ & Physical activity & Alcohol & $\begin{array}{l}\text { Dietary patterns and other behaviour } \\
\text { changes identified during the confinement }\end{array}$ \\
\hline 16 & $\begin{array}{l}\text { Gentile, et al., } \\
2020, \\
\text { Vasto-Italy } \\
\text { and } \\
\text { Paragua- } \\
\mathrm{y}^{(61)} \text {. }\end{array}$ & $4-6$ & $\begin{array}{l}\text { Provide psychiatric } \\
\text { assessments and } \\
\text { measure the level of } \\
\text { stress related to } \\
\text { quarantine in a large } \\
\text { sample of psychiat- } \\
\text { ric outpatients }\end{array}$ & 110 & $\uparrow 7.27 \%$ & NR & NR & NR & NR & $\uparrow 2.72 \%$ & $\begin{array}{l}56.3 \% \text { self- reported lifestyle changes dur- } \\
\text { ing the confinement including: } \\
32.7 \% \text { eating pattern changes, } 4.54 \% \\
\text { Change in sleeping pattern, increased alco- } \\
\text { hol in } 2.72 \% \\
\text { Consumption, more reading } \\
\text { and gaming in } 16.3 \% \text {. Self-reported emo- } \\
\text { tions from the patients ranked: } \\
\text { Fear } 24.5 \% \text { Optimism } 20 \% \text { Pessimism } \\
14.5 \% \text {, Hope } 13.6 \% \text {, Hopelessness } \\
10.9 \% \text {, Serenity, Anger } 7.27 \%\end{array}$ \\
\hline 17 & $\begin{array}{l}\text { Giustino et al., } \\
2020 \\
\text { Italy }\end{array}$ & $1-2$ & $\begin{array}{l}\text { Changes in physical } \\
\text { activity before and } \\
\text { during the quaran- } \\
\text { tine among the } \\
\text { active Sicilian popu- } \\
\text { lation }\end{array}$ & 802 & NR & $\begin{array}{r}\downarrow 1168.5 \mathrm{MET} \\
-\mathrm{min} / \text { week }\end{array}$ & NR & NR & NR & $N R$ & $\begin{array}{l}\text { Greater impact of decreased physical activ- } \\
\text { ity among males and overweight partici- } \\
\text { pants }\end{array}$ \\
\hline 18 & $\begin{array}{l}\text { He, et al., } \\
2020 \\
\text { China }^{(39)}\end{array}$ & 4 & $\begin{array}{l}\text { Body weight, physical } \\
\text { activity and lifestyle } \\
\text { changes }\end{array}$ & 339 & $\begin{array}{l}\text { BMl }<24 \text { gained } \\
\quad \text { weight } \\
\text { Females: } 2 \cdot 2 \mathrm{~kg} \\
\text { Males: } \\
1.7 \mathrm{~kg}\end{array}$ & Decreased & NR & NR & Decreased & Decreased & $\begin{array}{l}\text { Weight correlated with the change level of } \\
\text { alcohol consumption } \\
\text { during the semi-lockdown for COVID-19 } \\
(R s=-0.255 \\
P=0.002)\end{array}$ \\
\hline 19 & $\begin{array}{l}\text { Ismail, et al., } \\
\quad 2020, \\
\text { MENA } \\
\text { Region } \\
{ }^{(3)} .\end{array}$ & $4-6$ & $\begin{array}{l}\text { Eating behaviours and } \\
\text { lifestyle changes } \\
\text { during COVID - } 19 \\
\text { pandemic in Middle } \\
\text { east and North } \\
\text { Africa region } \\
\text { (MENA) }\end{array}$ & 2970 & $\uparrow 30.3 \%$ & Increased & $\begin{array}{l}32.9 \% \text { had salty } \\
\text { snacks }\end{array}$ & $\begin{array}{l}48.8 \% \text { of sur- } \\
\text { veyed partici- } \\
\text { pants did not } \\
\text { consume fruits } \\
\text { and } 32.5 \% \text { did } \\
\text { not consume } \\
\text { vegetables } \\
\text { daily }\end{array}$ & $\begin{array}{l}\text { Increased level of } \\
\text { inactivity from } \\
34.9 \% \text { to } \\
39.1 \%\end{array}$ & NR & $\begin{array}{l}\text { Skipping meals decreased } \\
74.0 \% \text { consumed less than eight cups of } \\
\text { water per day. } \\
44.1 \% \text { ate sweets or desserts }\end{array}$ \\
\hline 20 & $\begin{array}{l}\text { Ismail, et al., } \\
2020 \\
\operatorname{UAE}^{(33)}\end{array}$ & $1-4$ & $\begin{array}{l}\text { Effect of quarantine on } \\
\text { eating habits, physi- } \\
\text { cal activity, stress } \\
\text { and sleep behav- } \\
\text { iours }\end{array}$ & 1012 & $\begin{array}{l}\uparrow 31 \% \\
\downarrow 20.9 \%\end{array}$ & $\begin{array}{l}\uparrow 25.71 \% \\
\downarrow 12.31 \%\end{array}$ & $\begin{array}{l}37.1 \% \text { ate salty } \\
\text { snacks }\end{array}$ & $\begin{array}{l}48.8 \% \text { consumed } \\
\text { fruits daily }\end{array}$ & $\begin{array}{l}\uparrow 14.8 \\
\downarrow 41.9\end{array}$ & NR & $\begin{array}{l}\text { Increase in home cooked food, decrease in } \\
\text { fast food consumption }(P<0.0001) \text {. } \\
\text { Decrease frequency of meal skipping } \\
(64.5-46.2 \%) \text {. } \\
\text { Increase breakfast intake (66\% to } 74.2 \%) \text {. } \\
\text { Increase water intake ( } 24.1-27.8 \% \text {.) } \\
\text { Main products consumed are sweets and } \\
\text { desserts and salty snacks (chips, crack- } \\
\text { ers, and nuts) during COVID-19 pan- } \\
\text { demic } \\
\text { Inactivity levels rise (32.1-38.5\%). } \\
69.1 \text { and } 67.8 \% \text { of participants relied on } \\
\text { social media applications to be updated } \\
\text { about nutrition and health news }\end{array}$ \\
\hline 21 & $\begin{array}{l}\text { Jia, et al., } \\
\quad 2020, \\
\quad \text { China } \\
{ }^{(56)}\end{array}$ & $11-14$ & $\begin{array}{l}\text { Activity performance } \\
\text { and weight changes }\end{array}$ & 10082 & $\begin{array}{l}\text { BMl increased from } \\
21.8 \text { to } 22.1 \mathrm{~kg} / \mathrm{m}^{2} \\
\text { Overweight subject's } \\
\text { percentage } \\
\text { increased from } \\
21.4 \% \text { to } 24.6 \% \\
\text { Obesity participants } \\
\quad \text { percentage } \\
\text { increased from } \\
10.5 \% \text { to } 12.6 \%\end{array}$ & NR & NR & NR & $\begin{array}{l}\text { Decreased } \\
(1.3-0.9 \mathrm{~d} / \text { week } \\
\quad P<0.001)\end{array}$ & NR & $\begin{array}{l}\text { Increased sleeping hours }(7.4-7.6 \mathrm{~h} / \text { week, } \\
P<0.001) \text { on weekdays and }(7.9-8.0 \mathrm{~h} / \\
\text { week, } P<0.001) \text { on weekends } \\
\text { Increase sedentary lifestyle }(4.2-5.3 \mathrm{~h} / \\
\text { week, } P<0.001) \text { on weekdays and } \\
(4.3-5.1 \mathrm{~h} / \text { week, } P<0.001) \text { on } \\
\text { weekends } \\
\text { Increased screen time }(4.9-5.6 \mathrm{~h} / \text { week, } \\
P<0.001)\end{array}$ \\
\hline
\end{tabular}




\section{NS British Journal of Nutrition}

Table 3. (Continued)

\begin{tabular}{|c|c|c|c|c|c|c|c|c|c|c|c|}
\hline $\begin{array}{l}\text { S. } \\
\text { no }\end{array}$ & $\begin{array}{l}\text { First author, } \\
\text { year, country }\end{array}$ & $\begin{array}{l}\text { Duration of } \\
\text { confinement } \\
\text { during which } \\
\text { study was } \\
\text { conducted } \\
\text { (weeks) }\end{array}$ & Outcome area of focus & $\begin{array}{l}\text { Number of } \\
\text { participants }\end{array}$ & $\begin{array}{l}\text { Weight gain of the } \\
\text { participant }(\%) \\
\text { (weight gain in } \mathrm{kg} \text { ), } \\
\text { weight loss of the } \\
\text { participant }(\%) \\
\text { (weight loss in } \mathrm{kg} \text { ) }\end{array}$ & $\begin{array}{l}\text { Energy intake/ } \\
\text { food intake }\end{array}$ & Snacking & $\begin{array}{l}\text { Fresh product } \\
\text { (fruits and vegeta- } \\
\text { ble) }\end{array}$ & Physical activity & Alcohol & $\begin{array}{l}\text { Dietary patterns and other behaviour } \\
\text { changes identified during the confinement }\end{array}$ \\
\hline 22 & $\begin{array}{l}\text { Jimenez, et al., } \\
2021, \\
\text { Spain } \\
{ }^{(43)} \text {. }\end{array}$ & 9 & $\begin{array}{l}\text { Psychosocial, lifestyle } \\
\text { and body weight } \\
\text { effect due to } \\
\text { COVID-19 lockdown }\end{array}$ & 603 & $\uparrow 52 \cdot 2$ & Increased & $\uparrow 19 \%$ & $\uparrow 32.5 \%$ & $\begin{array}{l}\text { Decreased in }>50 \\
\quad \%\end{array}$ & $\begin{array}{l}\text { Almost unchanged in } \\
81.4 \% \\
(1.6 \pm 1.2 \mathrm{~V} \\
1.3 \pm 0.7 \\
P<0.01) \\
\end{array}$ & 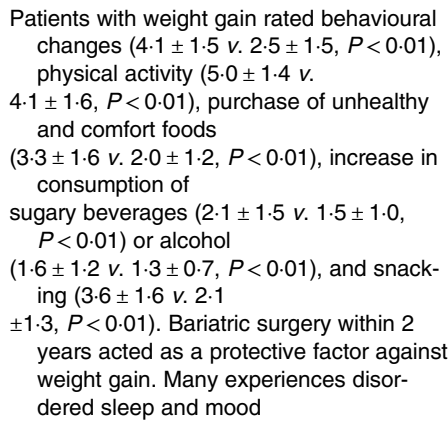 \\
\hline 23 & $\begin{array}{l}\text { Kang, et al., } \\
2021 \\
\text { Korea }^{(49)}\end{array}$ & 24 & $\begin{array}{l}\text { COVID-19 impact on } \\
\text { childhood obesity } \\
\text { and vitamin D levels }\end{array}$ & 226 & $\begin{array}{l}\text { Overweight or obesity } \\
\text { rate increased } \\
23.9-31.4 \% \\
(7.5 \% \text { increase })\end{array}$ & $\mathrm{NR}$ & NR & NR & $\begin{array}{l}\text { Decreased due to } \\
\text { school closure }\end{array}$ & NR & $\begin{array}{l}\text { BMI } z \text { scores increased by } 0.219 \\
(P<0.001) \\
\text { Increase level of TAG (105.8 mg/dL v. } 88.6 \\
\text { mg/dl, } P<0.001) \\
\text { Increase level of LDL-cholesterol }(100.2 \\
\text { mg/dl v. } 94.0 \mathrm{mg} / \mathrm{dl}, P=0.002) \text {. } \\
\text { Decrease level of calcidiol level }(18.9 \mathrm{mg} / \mathrm{dl} \\
v .23 .8 \mathrm{mg} / \mathrm{dl}, P<0.001) \text {. } \\
\text { Patients who were normal weight had } 9.9 \\
(P<0.001) \text { times risk of becoming over- } \\
\text { weight or obesity during epidemic }\end{array}$ \\
\hline 24 & $\begin{array}{l}\text { Karatas, et al., } \\
2020, \\
\text { Istanbu|(65). }\end{array}$ & 24 & $\begin{array}{l}\text { Body weight, metabolic } \\
\text { control in type } 2 \text { dia- } \\
\text { betic patient and } \\
\text { healthy population }\end{array}$ & 140 & $\begin{array}{l}\text { Non-diabetic group } \\
(86.10 \pm 10.48 \mathrm{v} \\
85.56 \pm 10.53 \mathrm{~kg}) \\
(P<0.05) \\
(0.54 \pm 0.95) \\
\text { Diabetic group } \\
(89.75 \pm 18.68 \mathrm{v} \\
87.83 \pm 18.27 \mathrm{~kg}) \\
(P<0.05) \\
1.91 \pm 5.48 \mathrm{~kg}\end{array}$ & NR & $\mathrm{NR}$ & NR & NR & NR & $\begin{array}{l}\text { Non-significant change of BMl } 33.44 \pm 6.48 \\
\text { to } 31.63 \pm 3.57 \mathrm{~kg} / \mathrm{m}^{2} \\
\text { HbA1c increased more in diabetic than in } \\
\text { non-diabetic groups }(P=0.002) \\
\text { Glucose, LDL-cholesterol, and TAG } \\
\text { increased in diabetic }(39.69 \pm 74.69, \\
7.60 \pm 34.33, \text { and } 58.21 \pm 133.54 \mathrm{mg} / \mathrm{dl}, \\
P<0.05) \\
\text { Waist circumference increased more in dia- } \\
\text { betic patients compared with non-dia- } \\
\text { betics }(1.20 \pm 2.38 \mathrm{v} .0 .03 \pm 0.46 \mathrm{~cm}, \\
P<0.05) \\
\text { TAG levels increased more in the diabetic } \\
\text { group than in the non-diabetic group } \\
(P=0.041)\end{array}$ \\
\hline 25 & $\begin{array}{l}\text { Keel PhD, } \\
\text { et al., 2020, } \\
\text { USA }^{(37)} \text {. }\end{array}$ & $6-7$ & $\begin{array}{l}\text { Perceived } v \text { observed } \\
\text { weight changes in } \\
\text { undergrad students } \\
\text { during COIVD-19 } \\
\text { confinements }\end{array}$ & 90 & $\begin{array}{l}\text { No statistically signifi- } \\
\text { cant }\end{array}$ & Increased & NR & NR & Decreased & NR & $\begin{array}{l}\text { Increase mean of weight description } \\
\text { Increase screen time } \\
\text { Increase time spent on TV } \\
\text { Increased weight/shape concerns were } \\
\text { significantly related to increased eating } \\
\text { concerns } \\
\text { Women had significantly higher weight/ } \\
\text { shape concerns than men } \\
\text { Women at time } 2 \text { spent more time on social } \\
\text { media compared with men }\end{array}$ \\
\hline
\end{tabular}


NS British Journal of Nutrition

Table 3. (Continued)

\begin{tabular}{|c|c|c|c|c|c|c|c|c|c|c|c|}
\hline $\begin{array}{l}\text { S. } \\
\text { no }\end{array}$ & $\begin{array}{l}\text { First author, } \\
\text { year, country }\end{array}$ & $\begin{array}{l}\text { Duration of } \\
\text { confinement } \\
\text { during which } \\
\text { study was } \\
\text { conducted } \\
\text { (weeks) }\end{array}$ & Outcome area of focus & $\begin{array}{l}\text { Number of } \\
\text { participants }\end{array}$ & $\begin{array}{l}\text { Weight gain of the } \\
\text { participant }(\%) \\
\text { (weight gain in } \mathrm{kg} \text { ), } \\
\text { weight loss of the } \\
\text { participant }(\%) \\
\text { (weight loss in } \mathrm{kg} \text { ) }\end{array}$ & $\begin{array}{l}\text { Energy intake/ } \\
\text { food intake }\end{array}$ & Snacking & $\begin{array}{l}\text { Fresh product } \\
\text { (fruits and vegeta- } \\
\text { ble) }\end{array}$ & Physical activity & Alcohol & $\begin{array}{l}\text { Dietary patterns and other behaviour } \\
\text { changes identified during the confinement }\end{array}$ \\
\hline 26 & $\begin{array}{l}\text { Kriaucioniene, } \\
\text { et al., 2020, } \\
\text { Lithuania(38). }^{(38} \text {. }\end{array}$ & 4 & $\begin{array}{l}\text { Effect of COVID-19 on } \\
\text { health behaviours } \\
\text { and body weight }\end{array}$ & 2447 & $\uparrow 31.5 \%$ & $\uparrow 49.4$ & $\uparrow 45.1 \%$ & $\begin{array}{l}\downarrow 14.7 \text { fruits } \\
\downarrow 15.0 \% \text { vegeta- } \\
\quad \text { bles }\end{array}$ & $\begin{array}{l}69.9 \% \text { remained } \\
\quad \text { the same } \\
\uparrow 14.2 \% \\
\downarrow 15.9 \%\end{array}$ & $\downarrow 60.6$ & $\begin{array}{l}62.1 \% \text { cooked at home more frequently } \\
\text { and }(37.7 \% \text { increased the intake of } \\
\text { homemade pastries while } 26 \% \\
\text { decreased intake of commercial pastries } \\
20.6 \% \text { ate more fried food } \\
41.3 \% \text { decreased fast food orders } \\
19.4 \% \text { decreased carbonated and sugary } \\
\text { drink intake }\end{array}$ \\
\hline 27 & $\begin{array}{l}\text { Malkawi, et al., } \\
2020, \\
\text { Jordann }\end{array}$ & $1-6$ & $\begin{array}{l}\text { Mental health and } \\
\text { changes in lifestyle } \\
\text { practices among } \\
\text { Jordanian mothers } \\
\text { during COVID-19 } \\
\text { quarantine }\end{array}$ & 2103 & $\uparrow 37 \%$ & NR & NR & $\begin{array}{l}80.7 \% \text { consumed } \\
\text { healthy diet }\end{array}$ & NR & NR & $\begin{array}{l}\text { Increased teaching time of children } \\
\text { Increased }(63 \%) \text {. } \\
\text { Family violence } \\
\text { Increased }(27 \%) \text { hours spent in dedicated } \\
\quad \text { family time }(+5 \mathrm{~h} \text { ). } \\
\text { Mild levels of depression (mean }=11.5 \pm \\
\quad \mathrm{SD}=9 \text {; } \\
\text { range } 0-42 \text { ), anxiety (mean }=7 \cdot 2 \pm \mathrm{SD}=4 \text {; } \\
\quad \text { range } 0-42 \text { ), and stress } \\
\text { (mean }=14.7 \pm \mathrm{SD}=10 \text {; range } 0-42 \text { ). }\end{array}$ \\
\hline 28 & $\begin{array}{l}\text { Marchitelli, } \\
\text { et al., 2020, } \\
\text { Italy }\left({ }^{(4)} \text {. }\right.\end{array}$ & $9-11$ & $\begin{array}{l}\text { Weight gain in over- } \\
\text { weight/obese sub- } \\
\text { jects } \\
\text { Effect of psychological } \\
\text { and psychosocial } \\
\text { variables }\end{array}$ & 110 & $\begin{array}{l}\text { Weight gain by } 31 \% \\
\text { of overweight/ } \\
\text { obesity } \\
\text { Weight gain by } 31 \% \\
\text { of psychiatric } \\
\text { patients }\end{array}$ & $\begin{array}{l}60 \% \\
\text { increased } \\
\text { night eating }\end{array}$ & $\begin{array}{l}\text { No significant } \\
\text { changes }\end{array}$ & NR & NR & NR & $\begin{array}{l}\text { Binge eating was significant factor for } \\
\text { weight gain in psychiatric patients } \\
\text { Increased night eating episodes in } \\
\text { response to stress }\end{array}$ \\
\hline 29 & $\begin{array}{l}\text { Martínez-de- } \\
\text { Quel et al., } \\
2021, \\
\text { Spain }{ }^{(45)}\end{array}$ & $6-7$ & $\begin{array}{l}\text { Changes in physical } \\
\text { activity, dietary hab- } \\
\text { its and sleep quality } \\
\text { pre- and post-lock- } \\
\text { down }\end{array}$ & 161 & $\begin{array}{l}\text { Pre } 67.3 \mathrm{~kg} \pm 14.8 \mathrm{~V} \\
\text { Post } 67.7 \mathrm{~kg} \\
\pm 15.1\end{array}$ & NR & NR & NR & $\begin{array}{l}\downarrow 8515 \cdot 7 \pm \\
10260.0 \text { Met/ week } \\
v \\
\text { Post } \\
5053 \cdot 5 \pm 5502 \cdot 0 \\
\text { Met/ week } \mathrm{p}= \\
<0.001\end{array}$ & NR & $\begin{array}{l}\text { Significant differences were found pre- and } \\
\text { post-lockdown with physical activity } \\
\text { sleep and } \\
\text { perceived well-being, } \\
\text { More people living together had higher } \\
\text { weight gain }\end{array}$ \\
\hline 30 & $\begin{array}{l}\text { Mason, et al., } \\
2021, \\
\text { USA } \\
(66) .\end{array}$ & 10-18 & $\begin{array}{l}\text { Body weight change } \\
\text { during lockdown and } \\
\text { factors determining } \\
\text { it }\end{array}$ & 1820 & $\begin{array}{l}\text { Mean weight change } \\
3.47 \mathrm{lbs}(\mathrm{sD} \\
14.57) ; \text { mean } \% \\
\text { Weight change } \uparrow \\
2.5 \%(8.6 \%)\end{array}$ & $\uparrow 31 \%$ & NR & NR & NR & NR & $\begin{array}{l}35 \% \text { consumed unhealthy food to cope with } \\
\text { the pandemic } \\
\text { Overeating as a mechanism of coping with } \\
\text { pandemic was related to increase in } \\
\text { weight and BMl on overweight }\end{array}$ \\
\hline 31 & $\begin{array}{l}\text { Mitchell et al., } \\
2020 \\
\text { USA }^{(21)} \text {. }\end{array}$ & 1 & $\begin{array}{l}\text { Alterations in food } \\
\text { choices related to } \\
\text { lockdown in users } \\
\text { enrolled in a digital } \\
\text { behavioural change } \\
\text { weight loss pro- } \\
\text { gramme }\end{array}$ & 381564 & NR & NR & NR & $\downarrow 4.2 \%$ & NR & $\downarrow 4.5 \%$ & $\begin{array}{l}\text { Use of the mobile app (Noom) decreased } \\
\text { by } 9 \%\end{array}$ \\
\hline 32 & $\begin{array}{l}\text { Önmez, et al., } \\
\text { 2020, } \\
\text { Turkey(64). }\end{array}$ & $15-24$ & $\begin{array}{l}\text { Glycaemic control in } \\
\text { type } 2 \text { diabetes } \\
\text { patients }\end{array}$ & 101 & $\begin{array}{l}\uparrow 39.6 \% \\
\ 38.6 \%,\end{array}$ & NR & NR & NR & $\begin{array}{l}\text { Low: } \\
\text { physical function- } \\
\text { ing on short } \\
\text { form } 36-\text { item } \\
\text { survey } \\
(59.5 \pm 26.9)\end{array}$ & NR & $\begin{array}{l}\text { HbA1c increased from } 7 \cdot 67 \pm 1 \cdot 76 \text { to } \\
8 \cdot 11 \pm 2 \cdot 48 \text { compared with pre- and post- } \\
\text { lockdown. The numbers of patients who } \\
\text { exercised regularly and dieted were low. } \\
\text { Mean pre-lockdown waist circumference } \\
\text { was } 105 \pm 23 \mathrm{~cm} \text {, compared with } \\
107 \pm 32 \mathrm{~cm} \text { post-lockdown }\end{array}$ \\
\hline
\end{tabular}


WS British Journal of Nutrition

Table 3. (Continued)

\begin{tabular}{|c|c|c|c|c|c|c|c|c|c|c|c|}
\hline $\begin{array}{l}\text { S. } \\
\text { no }\end{array}$ & $\begin{array}{l}\text { First author, } \\
\text { year, country }\end{array}$ & $\begin{array}{l}\text { Duration of } \\
\text { confinement } \\
\text { during which } \\
\text { study was } \\
\text { conducted } \\
\text { (weeks) }\end{array}$ & Outcome area of focus & $\begin{array}{l}\text { Number of } \\
\text { participants }\end{array}$ & $\begin{array}{l}\text { Weight gain of the } \\
\text { participant }(\%) \\
\text { (weight gain in } \mathrm{kg} \text { ), } \\
\text { weight loss of the } \\
\text { participant }(\%) \\
\text { (weight loss in kg) }\end{array}$ & $\begin{array}{l}\text { Energy intake/ } \\
\text { food intake }\end{array}$ & Snacking & $\begin{array}{l}\text { Fresh product } \\
\text { (fruits and vegeta- } \\
\text { ble) }\end{array}$ & Physical activity & Alcohol & $\begin{array}{l}\text { Dietary patterns and other behaviour } \\
\text { changes identified during the confinement }\end{array}$ \\
\hline 33 & $\begin{array}{l}\text { Özden, et al., } \\
\quad 2021, \\
\quad \text { Turkey } \\
\end{array}$ & $8-10$ & $\begin{array}{l}\text { Nutrition exercise } \\
\text { behaviours during } \\
\text { lockdown }\end{array}$ & 1011 & $\uparrow 46.9 \%$ & Increased & Increased & NR & $\downarrow 35.4$ & NR & $\begin{array}{l}26.8 \% \text { were bored. Psychological/addictive } \\
\text { eating behaviour subscale scores } \\
\text { were piled up between } 20 \text { and } 40 \text {, and their } \\
\text { unhealthy nutrition-exercise behaviour } \\
\text { subscale mean scores were piled up } \\
\text { between } 30 \text { and } 50 \text { (Fig. 1) }\end{array}$ \\
\hline 34 & $\begin{array}{l}\text { Pellegrini et al., } \\
2020 \\
\text { Italy(22). }\end{array}$ & 4 & $\begin{array}{l}\text { Weight and dietary } \\
\text { changes before and } \\
\text { during the COVID- } \\
19 \text { lockdown in } \\
\text { obese adults }\end{array}$ & 150 & $\uparrow 1.51 \mathrm{~kg}$ & $\uparrow 40 \%$ & $\uparrow 33 \%$ & $\downarrow 18 \%$ & $\downarrow 60 \%$ & NR & $\begin{array}{l}\text { Increased weight gain with lower educa- } \\
\text { tional attainment and unhealthy food } \\
\text { choices. Anxiety and depression } \\
\text { increased weight gain by an average of } \\
2.69 \mathrm{~kg}(95 \% \mathrm{Cl} 1.66,3.71 ; P<0.001)\end{array}$ \\
\hline 35 & $\begin{array}{l}\text { Pietrobelli } \\
\text { et al., 2020, } \\
\text { Italy(23). }\end{array}$ & 3 & $\begin{array}{l}\text { Impact of COVID-19 } \\
\text { lockdown on lifestyle } \\
\text { factors in obese chil- } \\
\text { dren }\end{array}$ & 41 & NR & $\begin{array}{l}\uparrow 1.15 \pm 1.56 \\
\text { meals per } \\
\text { day }\end{array}$ & NR & NR & $\begin{array}{l}\downarrow 2 \cdot 30 \pm 4.60 \mathrm{~h} / \\
\text { week }\end{array}$ & NR & $\begin{array}{l}\text { Unhealthy food intake increased with signifi- } \\
\text { cantly increased potato chips, red meat } \\
\text { and sugary drink intakes during the lock- } \\
\text { down }(0.005 \text { to }<0.001) \\
\text { Screen time increased by } 4.85 \pm 2.40 \mathrm{~h} / \mathrm{d} \\
\text { Sleep time increased by } 0.65 \pm 1.29 \mathrm{~h} / \mathrm{d}\end{array}$ \\
\hline 36 & $\begin{array}{l}\text { Rogers et al., } \\
2020 \\
\text { UK } \\
\end{array}$ & $2-4$ & $\begin{array}{l}\text { Altered physical activity } \\
\text { among adults with seri- } \\
\text { ous health issues } \\
\text { during COVID-19 } \\
\text { lockdown }\end{array}$ & 5820 & NR & NR & NR & NR & $\uparrow 11.7 \%, \downarrow 25.4 \%$ & NR & $\begin{array}{l}\text { Being a female, living alone or not having } \\
\text { access to a garden were also associated } \\
\text { with less intensive physical activity }\end{array}$ \\
\hline 37 & $\begin{array}{l}\text { Romero- } \\
\text { Blanco } \\
\text { et al., 2020, } \\
\text { Spain } \\
{ }^{62)} \text {. }\end{array}$ & 4 & $\begin{array}{l}\text { Sleep quality before } \\
\text { and during the } \\
\text { COVID-19 lockdown } \\
\text { period in nursing } \\
\text { students }\end{array}$ & 207 & NR & NR & NR & NR & NR & NR & $\begin{array}{l}\text { Pittsburgh sleep quality } \\
\text { index (PSQI) scored } 0.91 \text { points worse dur- } \\
\text { ing the lockdown. Poor sleep incidence } \\
\text { increased from } 60.4 \% \text { to } 67.1 \% \text { during } \\
\text { the lockdown. Students with anxiety and } \\
\text { depression had reduced sleep quality by } \\
1.74(0.85-2.63) \text { points }\end{array}$ \\
\hline 38 & $\begin{array}{l}\text { Ruissen, et al., } \\
\text { 2021, } \\
\text { Netherland- } \\
\mathrm{s}^{(59)} \text {. }\end{array}$ & $8-11$ & $\begin{array}{l}\text { Lockdown impact on } \\
\text { people with type } 1 \\
\text { and type } 2\end{array}$ & 435 & $\begin{array}{l}\uparrow 40.9 \% \\
\downarrow 12 \%\end{array}$ & NR & NR & NR & $\downarrow 45.7 \%$ & NR & $\begin{array}{l}\text { Increase in levels of stress } 34.1 \% \\
\text { Increase in levels of anxiety } 27.3 \% \text { of all } \\
\text { participants } \\
\text { Stress correlated with poor glycaemic con- } \\
\text { trol }(P<0.0001)\end{array}$ \\
\hline 39 & $\begin{array}{l}\text { Shah, et al., } \\
2020 \\
\text { India }^{(47)}\end{array}$ & $\begin{array}{l}12-15 \\
\text { weeks }\end{array}$ & $\begin{array}{l}\text { Glycaemic control, } \\
\text { weight and BMI }\end{array}$ & 77 & $\begin{array}{l}\text { Weight gain } z \text { score } \\
-0.4 \pm 0.8 \mathrm{v} \text {. Post- } \\
\text { lockdown weight } z \\
\text { score }-0.2 \pm 0.8 \\
P<0.05) \\
\text { No significant } \\
\text { increase in BMI }\end{array}$ & $\begin{array}{l}\downarrow \text { in low socio- } \\
\text { economic } \\
\text { state }\end{array}$ & Decreased & NR & NR & NR & $\begin{array}{l}\text { Improved glycaemic control via HbA1C } \\
79.4 \pm 19.2 \mathrm{v} \text {. Post-lockdown Hba1C } \\
74.5 \pm 16.9 \mathrm{mmol} / \mathrm{mol} \\
\text { Improved glycaemic control in lower socio- } \\
\text { economic state }\end{array}$ \\
\hline 40 & $\begin{array}{l}\text { Sidor et al., } \\
\quad 2020 \\
\text { Poland } \\
(24)\end{array}$ & 6 & $\begin{array}{l}\text { Sleep quality before } \\
\text { and during the } \\
\text { COVID-19 lockdown } \\
\text { period in nursing } \\
\text { students }\end{array}$ & 1097 & $\begin{array}{l}\uparrow 29.9 \%(3.0 \pm 1.6 \\
\mathrm{kg}), \downarrow 18.6 \% \\
(2.9 \pm 1.5 \mathrm{~kg})\end{array}$ & $\uparrow 43.5 \%$ & $\uparrow 51.8 \%$ & NR & NR & $\uparrow 14.6 \%$ & $\begin{array}{l}\text { Increased food consumption }(55.3 \%) \text { and } \\
\text { snacking }(61.7 \%) \text { was reported by indi- } \\
\text { viduals with a higher BMI }\end{array}$ \\
\hline 41 & $\begin{array}{l}\text { Zachary et al., } \\
2020 \\
\text { USA }^{(25)}\end{array}$ & 4 & $\begin{array}{l}\text { Diet choices and habits } \\
\text { during COVID-19 } \\
\text { lockdown }\end{array}$ & 173 & $\uparrow 22 \%$ & $\uparrow 19 \%$ & $\uparrow 63 \%$ & NR & NR & NR & $\begin{array}{l}73 \% \text { ate in response to boredom and } 65 \% \\
\text { in response to sight/smell of food } \\
\text { Participants slept an average of } 7.6 \pm 1.3 \mathrm{~h} \\
\text { per night with less sleep related to weight } \\
\text { gain }\end{array}$ \\
\hline
\end{tabular}

$\uparrow$, increased; $\downarrow$, decreased; NR, not reported; MET-minute/week, metabolic equivalent of task minute/week; IQR, interquartile range; Ibs, pound. 
Table 4. Determinants of body weight during pandemic confinements

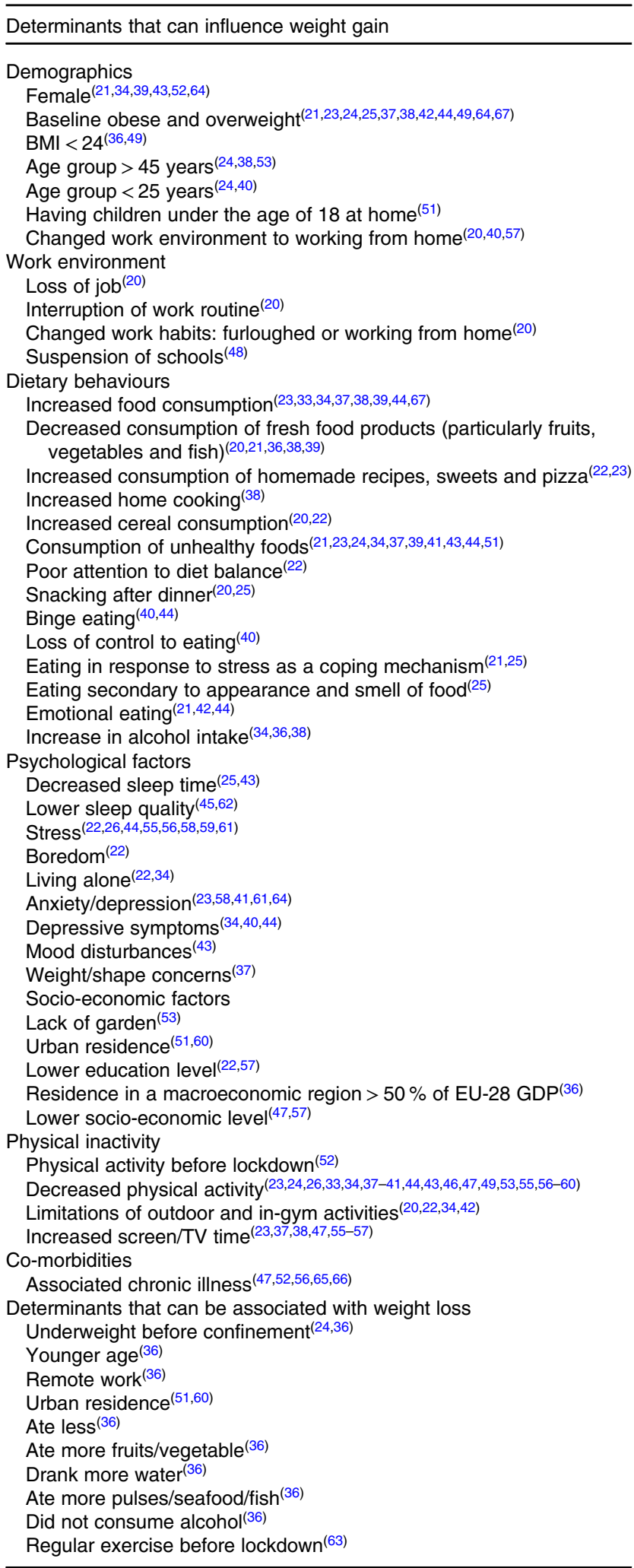

this current systematic review. This includes past behaviours, dietary behaviours, physical activity patterns, work environment, psychosocial and socio-economic factors, and preexisting co-morbidities.
Female sex ${ }^{(20,34,38,42,51,63)}$, age under 25 years and over 45 years $^{(24,38,51,53)}$ are in particular at higher risk of gaining weight. Initial weight status, diet quality and physical exercise pattern before lockdown are important factors ${ }^{(20,22-24,36,44,48,51,63)}$. In Chinese $^{(39)}$ and Korean ${ }^{(49)}$ populations, BMI $<24 \mathrm{~kg} / \mathrm{m}^{2}$ was associated with weight gain. However, some observed that those who were underweight before confinement lost more weight during confinement ${ }^{(24,36)}$.

Poor diet quality before the lockdown was associated with weight gain ${ }^{(51)}$. Decreased consumptions of legumes, fruits and vegetables ${ }^{(24,38)}$ were related to an increased consumption of sweets ${ }^{(22-24)}$. Moreover, more home cooking with consumption of unhealthy foods is associated with increased weight gain $^{(19,21,22,33-35,37,39,41,42,47,64)}$ as is increased alcohol intake ${ }^{(34,36,38,46,67)}$

Less intense physical behaviours were noted during lockdown periods compared with behaviours before lockdown causing increased weight gain ${ }^{(22,23,25,32-34,36-40,42,45,46,48,52,54-59)}$. This was due to the limitations of outdoor activities and ingym activities ${ }^{(20,42,52)}$. In addition, there has been more sedentary behaviour with increased screen time ${ }^{(22,36,37,46)}$ which has been associated with weight gain.

Changed working habits, whether furloughed or working from home during the lockdown or those who had their job suspended $^{(20,51,57)}$, having children aged $<18$ years at home ${ }^{(51)}$, urban residence and attaining a lower educational level ${ }^{(22)}$ were associated with weight gain.

Patients with pre-existing psychiatric co-morbidities had weight gain during COVID-19 lockdown ${ }^{(34,37,43,44,51,61)}$, and $\operatorname{stress}^{(21,25,44,54,55,55,57,60,61)}$, anxiety and/or depression ${ }^{(22,57,60,61)}$, eating in response to $\operatorname{stress}^{(21,25)}$, boredom ${ }^{(22)}$, living alone ${ }^{(22)}$, emotional eating $^{(21,42,44)}$ or weight or body shape concerns ${ }^{(37)}$ were associated with an increase in body weight during confinement. Decreased sleeping time ${ }^{(25)}$ or poor quality sleep ${ }^{(45,50,62)}$ was further associated with weight gain.

Socio-economic factors such as urban residence ${ }^{(51,60)}$, lack of access to garden ${ }^{(53)}$, lower socio-economic level ${ }^{(47)}$ or lower education levels ${ }^{(22)}$ and residence in a macroeconomic region ${ }^{(36)}$ were associated with significant gain in the weight.

Patients with chronic illness such as diabetes, hypertension, lung disease, chronic CHD, congestive heart failure, depression or disability affecting one or more activities of daily living or lower levels of physically activity had an increase in weight $^{(34,40,44,46,51,53,55,61,64)}$.

Those who were previously underweight before the lockdown tended to lose more weight ${ }^{(24,24,36)}$. Those whose diet included more fruits and vegetables, pulses and drank more water lost weight ${ }^{(36)}$.

\section{Discussion}

This systematic review highlights contrasting effects of pandemic confinements on body weight, and we identified specific factors associated with change in body weight during the lockdown periods.

A BMI of $>25 \mathrm{~kg} / \mathrm{m}^{2}$ was identified as an independent risk factor for increased food intake during lockdown ${ }^{(68)}$. Other influences were inadequate sleep, decreased physical activity, 
emotional eating in response to stress, lack of control in dietary habits $(20,24,53)$ and increased alcohol consumption and smoking $(34,36,38,50,55,63,67)$. The impact of these influences is more significant in the obese population.

Eating habits as well as diet composition are linked to weight gain $^{(69)}$. Increased snacking after meals, particularly post dinner, was associated with weight gain ${ }^{(69)}$. Jakubowicz et al. also concluded that increased energy content at dinner increased the subjects' weight ${ }^{(70)}$. Thus, decreasing food consumption during and post dinner should be recommended.

Social networks, neighbourhood social activities and physical activity can influence an individual's opportunity to make better choices contributing to protection from obesity ${ }^{(71)}$. The absence of these influences during extended lockdown periods may facilitate a more obesogenic environment, thus encouraging weight gain $^{(72)}$.

By contrast, not all effects of pandemic confinement resulted in weight gain. In an Italian study, $38 \%$ of participants adhered to a Mediterranean diet. This may have been assisted by the Italian Ministry of Health publishing online materials regarding favourable lifestyle choices during the lockdown in April 2020 and providing practical guidelines on healthy behaviours ${ }^{(73,74)}$.

Pandemic confinements undoubtedly increase stress $^{(33,55,58-60)}$, 73 and $83 \%$ of respondents experienced an increase in anxiety and depression, respectively, with $70 \%$ reporting weight management issues, stock-piling food and stress eating ${ }^{(22,40,75)}$. Weight loss was reported in three studies by $13-19 \%$ of participants ${ }^{(20,24,51)}$. Two studies showed stress-related weight among working professionals and university students ${ }^{(76,77)}$. The mechanism is twofold and results from decreased, unchanged or increased energy intake coupled with adaptive adrenergic stimulated thermogenesis involving brown adipose tissues ${ }^{(78)}$. The weight loss observed in this systematic review may also be attributed to the negative effect of stress ${ }^{(20,24,25,51,79)}$.

The link between weight changes and stress has been studied extensively ${ }^{(80,81)}$. Behavioural and physiological explanations suggest that the sensation of eating is associated with a psychological escape from emotional distress ${ }^{(82)}$ and that the consumption of high energy foods alleviates stress ${ }^{(80)}$. During a pandemic, where cities and even entire nations were locked down, fear and anxiety related to COVID-19 induced an over eating behaviour. However, management of this associated condition is difficult ${ }^{(83)}$. The adverse effects of lockdown on the psychological and social well-being of society emphasise the need for strong public health interventions to support particularly at-risk people.

The associations between health outcomes, exercise and physical activity are well-established. The results from studies that we included in this review were mixed; some participants engaged in increased physical activity, while others had lower levels of physical activity. Confinement did not induce many sedentary participants to increase their physical activity. Other unhealthy behaviours such as increased screen time were noted which are similar to previous studies ${ }^{(84)}$. Stress may impair efforts to become physically active; conversely, those who already participate may do so to reduce stress ${ }^{(85)}$, which may explain the variation in physical activity observed. Seigel et al. describe this as stress-related behavioural activation or inhibition ${ }^{(86)}$.
Other unhealthy behaviours were noted during the confinement. There was a $14.6 \%$ increase in the consumption of alcohol in participants who had issues with alcohol ${ }^{(24)}$. In the acute postdisaster period of the September 11 attacks in Manhattan, New York City, the prevalence of alcohol consumption and marijuana use among New York City residents increased over a 5-8-week period $^{(87)}$. These results mirror our findings, suggesting shared responses to intense community stresses. Although these activities may not directly affect weight, alcohol consumption and obesity are common risk factors for chronic illnesses leading to increased morbidity and mortality ${ }^{(88)}$. Furthermore, in a study conducted in the Netherlands, it was reported that overweight and obese individuals found it more difficult to make healthy food choices. More savoury snacks and non-alcoholic beverages were purchased and consumed at home $(35.6 \%)$ because of more leisure time $(31.5 \%)$ and boredom (21.9\%) during the lockdown ${ }^{(89)}$

Positive outcomes from confinement have also been reported ${ }^{(90)}$. These behaviours may result from the increased availability of time to cook, health risk perceptions, lack of negative social distractions ${ }^{(91)}$ and socio-cognitive ideation towards a healthier lifestyle ${ }^{(92)}$. Long-term studies are necessary to determine whether these constructive and preventive behaviours can be sustained after confinement is over.

Food security, which involves food availability, accessibility and affordability, is another important factor in the relationship between pandemic confinement and body weight changes ${ }^{(93)}$. Global non-pharmaceutical interventions, such as lockdowns and quarantines, implemented to limit the spread of the virus have seriously impacted food security systems ${ }^{(94)}$, with the greatest burden affecting communities in which nutritional health is fragile ${ }^{(95)}$. Communities with precarious budgeting practices were destabilised by food price inflation and product shortages. Additional influences on food security included movement restrictions of workers, changes in consumer demand, closure of food production facilities, restricted food trade policies and financial pressures in the food supply chain. As dependence on food banks grew with an exponential increase in demand, basic survival needs presided over healthy dietary choices ${ }^{(17)}$. Prior to 2020, 690 million people were already food insecure and hungry ${ }^{(96)}$. By the end of 2020, the COVID-19 pandemic had created an additional 270 million food-insecure people ${ }^{(97,98)}$. Unfortunately, vulnerable populations are not restricted to under-resourced countries; developed nations are suffering as well. In the USA alone, food insecurity more than doubled as a result of the economic crisis brought on by the outbreak, impacting as many as $23 \%$ of households ${ }^{(99)}$.

Serious ethical and health-related issues hinder healthcare providers working with vulnerable populations. In general, differences in weight status and dietary intake reveal that a trend in obesity increases as the degree of food insecurity increases $^{(100)}$. The COVID-19 crisis has highlighted food insecurity as a significant factor in nutritional poverty ${ }^{(94)}$. This awareness of food insecurity may provide nations with the impetus to robustly tackle food-related epidemics, such as obesity and diabetes.

COVID-19 has challenged us to consider the role and balance of healthcare, personal health and holistic well-being. 
Redefining these dynamics in preparation for future pandemics is imperative to minimise severe impacts to health and resources $^{(101)}$. It was previously observed that consumerism is affected by internal factors, such as personal character, and external factors, such as economic crises. The pandemic served as an external factor that altered consumer behaviour $^{(102)}$.

Relief efforts by governmental and non-governmental agencies achieved temporary solutions without significant public pressure $^{(103)}$, but the demand for aid from all sectors of society is mounting. National governments should take the lead in providing strategic directions that will ensure the continuity of food accessibility to all, particularly the most vulnerable. Focus must be on coordinated and integrated public health programmes through legislative action to end sub-standard dietary conditions endured by those most in need. By collaborating with key stakeholders, health professionals must provide aggressive nutritional counseling to improve dietary habits, and concerted efforts across the board are paramount.

Recent research has shown obesity to be an independent risk factor for severe complications and increased mortality from COVID-19 ${ }^{(104,105)}$. The evidence suggests a linear relationship with obesity increasing the risk of severe disease and death among COVID-19 patients ${ }^{(106)}$. The co-existence of both pandemics, COVID-19 and obesity, along with the emergence of obesity evolving from lockdown has caused a 'syndemic' or a symbiotic pandemic ${ }^{(107)}$. Researchers must address the significant knowledge gaps that have become apparent during this pandemic regarding preparedness and response to such a crisis. Moreover, COVID-19 has disproportionately affected certain populations, and future research should focus on such vulnerable populations to ensure better outcomes.

\section{Strengths and limitations}

To our knowledge, this is the first systematic review evaluating the effects of pandemic confinement on body weight. Our study highlights major determinants that can have an impact on body weight during confinement and those that can be targeted in future pandemics to effectively manage body weight during pandemics via public health initiatives. Moreover, confinements are not solely related to pandemics and can also occur during natural disasters or calamities and in prisons. Determinants identified could be modified via appropriate public health measures to reduce negative impacts.

The present study has limitations. First, there was limited evidence from past pandemics related to obesity and morbidity or mortality. This may reflect the recent evolution of worldwide obesity $^{(108)}$. Second, within the common research theme of body weight changes during pandemic confinements, our systematic review found marked heterogeneity in the determinants and measured outcomes. This variation could be explained by differences in the study population and types of outcome measurements ${ }^{(109)}$. Nevertheless, in our systematic review, we followed a rigorous protocol with clear objectives and inclusion and exclusion criteria. This allowed for the identification and pooling of the determinants of body weight changes during pandemic confinements (Table 4). A thorough and complete identification of the different determinants related to pandemic confinements could guide decision makers. Furthermore, our study calls for further research into the level of impact of each determinant. Third, given the contemporary nature of the pandemic, the literature was primarily related to countries where COVID-19 had an early 'first wave' impact. Findings from other continents, particularly from Africa and South America, are yet to emerge. Fourth, online surveys using social media platforms were the predominant data collection method, which has recognised strengths and biases. Although the researchers used this form of data collection to reach a wider population, the likelihood of a bias towards a younger population should be noted. Fifth, although this analysis provides evidence for the effects of confinement on body weight, we are unable to comment on the potential for interventions such as lifestyle changes to attenuate the phenomenon. Sixth, because of the limited number of studies included, we were unable to correct for influences, such as pre-existing diets, and could not quantify the impact of possible factors in isolation. Although we know that weight gain is likely during confinement, further research using more sophisticated data collection techniques is necessary to determine the holistic impact of confinement to provide evidencebased practical solutions for future eventualities.

\section{Conclusion}

This systematic review highlights the significant effects that pandemic confinements can have in the short term on body mass. Poor sleep, snacking post dinner, lack of dietary restraint, preexisting overweight status, emotional eating due to stress and decreased physical activity are risk factors for weight gain.

Preparing for the next 'wave' is challenging given the multitude of factors that must be tailored to the local situations and available resources. Planning for future episodes requires a strong, evidence-based national policy in conjunction with clear guidelines to ensure that the negative sequelae of lockdowns are minimised.

\section{Acknowledgements}

We thank Gamila Hassan at the National Medical Library at UAEU for her strategic support in locating and uploading full-text articles to Covidence.

The authors received no specific funding for this work.

M. A. B. K., P. M., R. G., L. Ö. and H. M. formulated the research question and designed the study. M. A. B. K., P. M., R. G., K. K. A. and A. M. B. A. S. extracted and reviewed the data independently. L. Ö. and M. A. B. K. performed the literature search. M. A. B. K., P. M., R. G., A. M. B. A. S. and J. N. performed the literature review and data analysis. M. A. B. K., P. M., K. K. A., H. M., R. G, A. M. B. A. S., J. N., L. Ö., J. E. M. S. and J. K. contributed to drafting the paper. M. A. B. K., R. G., P. M., A. M. B. A. S. and K. K. A. equally contributed to all of the work as co-first authors.

We declare that there are no conflicts of financial and commercial interest that could be perceived as prejudicing the impartiality of the present study. 
This article was not plagiarised and had not previously been published in other journals.

\section{Supplementary material}

For supplementary material/s referred to in this article, please visit https://doi.org/10.1017/S0007114521000921

\section{References}

1. CDC COVID-19 Response Team (2020) Severe outcomes among patients with coronavirus disease 2019 (COVID-19) United States, February 12-March 16, 2020. MMWR Morb Mortal Wkly Rep 69, 343-346.

2. Madhav N, Oppenheim B, Gallivan M, et al. (2017) Pandemics: Risks, Impacts, Mitigation. Disease Control Priorities: Improving Health, Reducing Poverty, 3rd ed. Washington, DC: The International Bank for Reconstruction and Development/The World Bank.

3. Ismail L, Materwala H, Znati T, et al. (2020) Tailoring time series models for forecasting coronavirus spread: case studies of 187 countries. Comput Struct Biotechnol J 18, 2972-3206.

4. Khan MA \& Moverley Smith JE (2020) "Covibesity," a new pandemic. Obesity Med 19, 100282.

5. Al Falasi RJ \& Ab Khan M (2020) The impact of COVID-19 on Abu Dhabi and its primary care response. Aust journal general practice $\mathbf{4 9}$

6. Every-Palmer S, Jenkins M, Gendall P, et al. (2020) Psychological distress, anxiety, family violence, suicidality, and wellbeing in New Zealand during the COVID-19 lockdown: a cross-sectional study. PLoS One 15, e0241658.

7. Mozaffarian D, Hao T, Rimm EB, et al. (2011) Changes in diet and lifestyle and long-term weight gain in women and men. N Engl J Med 364, 2392-2404.

8. Khan MA, Hashim MJ, Mustafa H, et al. (2020) Global epidemiology of ischemic heart disease: results from the global burden of disease study. Cureus 12, e9349.

9. Gallo L, Moritz K \& Akison L (2020) Nutrient intake, physical activity levels, and metabolic status in Australian university biomedical students. Curr Dev Nutr 4, 1404-1404.

10. Tsenoli M, Moverley Smith JE \& Khan MA (2021) A community perspective of COVID-19 and obesity in children: causes and consequences. Obesity Med 22, 100327.

11. Diaz-Zavala RG, Castro-Cantú MF, Valencia ME, et al. (2017) Effect of the holiday season on weight gain: a narrative review. J Obes 2017, 2085136.

12. Rundle AG, Park Y, Herbstman JB, et al. (2020) COVID-19Related school closings and risk of weight gain among children. Obesity 28, 1008-1009.

13. Mason F, Farley A, Pallan M, et al. (2018) Effectiveness of a brief behavioural intervention to prevent weight gain over the Christmas holiday period: randomised controlled trial. BMJ 363, k4867.

14. Hafner JW, Hough SM, Getz MA, et al. (2010) All-terrain vehicle safety, use patterns in Central Illinois youth. J Rural Health 26, 67-72.

15. Pearl RL (2020) Weight Stigma and the 'Quarantine-15'. Obesity 28, 1180-1181.

16. Laborde D, Martin W, Swinnen J, et al. (2020) COVID-19 risks to global food security. Science 369, 500-502.

17. Huizar MI, Arena R \& Laddu DR (2020) The global food syndemic: the impact of food insecurity, Malnutrition and obesity on the healthspan amid the COVID-19 pandemic. Prog Cardiovasc Dis 64, 105-107.
18. The Lancet Global Health (2020) Food insecurity will be the sting in the tail of COVID-19. Lancet Glob Health $\mathbf{8}$, e737-e737.

19. Cuschieri S \& Grech S (2020) Obesity population at risk of COVID-19 complications. Glob Health Epidemiol Genom 5, e6.

20. Di Renzo L, Gualtieri P, Pivari F, et al. (2020) Eating habits and lifestyle changes during COVID-19 lockdown: an Italian survey. J Translational Med 18, 1-15.

21. Mitchell ES, Yang Q, Behr H, et al. (2020) Self-reported food choices before, during COVID-19 lockdown. MedRxiv. doi: 10.1101/2020.06.15.20131888

22. Pellegrini M, Ponzo V, Rosato R, et al. (2020) Changes in weight and nutritional habits in adults with obesity during the 'lockdown' period caused by the COVID-19 virus emergency. Nutrients 12, 2016.

23. Pietrobelli A, Pecoraro L, Ferruzzi A, et al. (2020) Effects of COVID-19 lockdown on lifestyle behaviors in children with obesity living in Verona, Italy: a longitudinal study. Obesity 28, 1382-1385.

24. Sidor A \& Rzymski P (2020) Dietary choices and habits during COVID-19 Lockdown: experience from Poland. Nutrients 12, 1657.

25. Zachary Z, Brianna F, Brianna L, et al. (2020) Self-quarantine, weight gain related risk factors during the COVID-19 pandemic. Obes Res Clin Pract 14, 210-216.

26. Higgins JP, Thomas J, Chandler J, et al. (2019) Cochrane Handbook for Systematic Reviews of Interventions. Hoboken, NJ: John Wiley \& Sons.

27. Moher D, Liberati A, Tetzlaff J, et al. (2009) Preferred reporting items for systematic reviews and meta-analyses: the PRISMA statement. Ann Intern Med 151, 264-269.

28. Zhang J, Zhang Y, Huo S, et al. (2020) Emotional eating in pregnant women during the COVID-19 pandemic and its association with dietary intake and gestational weight gain. Nutrients 12, 2250.

29. Herzog R, Álvarez-Pasquin MJ, Díaz C, et al. (2013) Are healthcare workers' intentions to vaccinate related to their knowledge, beliefs and attitudes? A systematic review. BMC Public Health 13, 154.

30. Modesti PA, Reboldi G, Cappuccio FP, et al. (2016) Panethnic differences in blood pressure in Europe: a systematic review and meta-analysis. PLoS One 11, e0147601.

31. Peterson J, Welch V, Losos M, et al. (2011) The NewcastleOttawa Scale (NOS) for Assessing the Quality of Nonrandomised Studies in Meta-Analyses. Ottawa: Ottawa Hospital Research Institute.

32. Cheikh Ismail L, Osaili TM, Mohamad MN, et al. (2020) Eating habits and lifestyle during COVID-19 lockdown in the United Arab Emirates: a cross-sectional study. Nutrients 12, 3314.

33. Ismail LC, Osaili TM, Mohamad MN, et al. (2020) Assessment of eating habits, lifestyle during coronavirus pandemic in the MENA region: a cross-sectional study. Br J Nutr 1-30.

34. Di Santo SG, Franchini F, Filiputti B, et al. (2020) The effects of COVID-19, quarantine measures on the lifestyles, mental health of people over 60 at increased risk of dementia. Front Psychiatr 11, 578628.

35. Dondi A, Candela E, Morigi F, et al. (2021) Parents' perception of food insecurity and of its effects on their children in Italy six months after the COVID-19 pandemic outbreak. Nutrients 13, 121.

36. Drywień ME, Hamulka J, Zielinska-Pukos MA, et al. (2020) The COVID-19 pandemic lockdowns and changes in body weight among polish women. A cross-sectional online survey Plifecovid-19 study. Sustainability 12, 7768. 
37. Keel PK, Gomez MM, Harris L, et al. (2020) Gaining "The Quarantine 15:" Perceived versus observed weight changes in college students in the wake of COVID-19. Int J Eating Disorders 53, 1801-1808.

38. Kriaucioniene V, Bagdonaviciene L, Rodríguez-Pérez C, et al. (2020) Associations between changes in health behaviours and body weight during the COVID-19 quarantine in Lithuania: the Lithuanian COVIDiet Study. Nutrients 12, 3119.

39. He M, Xian Y, Lv X, et al. (2020) Changes in body weight, physical activity, and lifestyle during the semi-lockdown period after the outbreak of COVID-19 in China: an online survey. Disaster Med Public Health Prep 1-6.

40. Athanasiadis DI, Hernandez E, Hilgendorf W, et al. (2020) How are bariatric patients coping during the coronavirus disease 2019 (COVID-19) pandemic? Analysis of factors known to cause weight regain among postoperative bariatric patients. Surg Obes Reltd Dis 17, 756-764.

41. Błaszczyk-Bębenek E, Jagielski P, Bolesławska I, et al. (2020) Nutrition Behaviors in Polish Adults before and during COVID-19 Lockdown. Nutrients 12, 3084

42. Ozden G \& Parlar Kiliç S (2021) The effect of social isolation during COVID-19 pandemic on nutrition and exercise behaviors of nursing students. Ecol Food Nutr 1-19.

43. Jimenez A, de Hollanda A, Palou E, et al. (2021) Psychosocial, lifestyle, body weight impact of COVID-19-Related lockdown in a sample of participants with current or past history of obesity in Spain. Obes Surg 1-10.

44. Marchitelli S, Mazza C, Lenzi A, et al. (2020) Weight gain in a sample of patients affected by overweight/obesity with and without a psychiatric diagnosis during the Covid-19 lockdown. Nutrients $\mathbf{1 2}, 3525$.

45. Martínez-de-Quel Ó, Suárez-Iglesias D, López-Flores M, et al (2021) Physical activity, dietary habits and sleep quality before and during COVID-19 lockdown: a longitudinal study. Appetite 158, 105019.

46. Chagué F, Boulin M, Eicher J-C, et al. (2020) Impact of lockdown on patients with congestive heart failure during the coronavirus disease 2019 pandemic. ESC Heart Fail 7, 4420-4423.

47. Shah N, Karguppikar M, Bhor S, et al. (2020) Impact of lockdown for COVID-19 pandemic in Indian children, youth with type 1 diabetes from different socio-economic classes. I Pediatr Endocrinol Metab 1.

48. Adıbelli D \& Sümen A (2020) The effect of the coronavirus (COVID-19) pandemic on health-related quality of life in children. Children Youth Serv Rev 119, 105595.

49. Kang HM, Jeong DC, Suh B-K, et al. (2021) The impact of the Coronavirus Disease-2019 pandemic on childhood obesity, vitamin D status. J Korean Med Sci 36, e21.

50. Dragun R, Veček NN, Marendić M, et al. (2021) Have Lifestyle Habits and Psychological Well-Being Changed among Adolescents and Medical Students Due to COVID-19 Lockdown in Croatia?. Nutrients 13, 97.

51. Deschasaux-Tanguy M, Druesne-Pecollo N, Esseddik Y, et al. (2020) Diet, physical activity during the COVID-19 lockdown period (March-May 2020): results from the French NutriNetSante cohort study. Am J Clin Nutr 113, 924-938.

52. Giustino V, Parroco AM, Gennaro A, et al. (2020) Physical activity levels and related energy expenditure during COVID-19 quarantine among the Sicilian active population: a cross-sectional online survey study. Sustainability 12, 4356

53. Rogers N, Roberts C, Waterlow N, et al. (2020) Behavioural change towards reduced intensity physical activity is disproportionately prevalent among adults with serious health issues or self-perception of high risk during the UK COVID-19 lockdown. Front Public Health 8, 575091.
54. Chopra S, Ranjan P, Singh V, et al. (2020) Impact of COVID-19 on lifestyle-related behaviours-a cross-sectional audit of responses from nine hundred and ninety-five participants from India. Diabetes Metab Syndrome: Clin Res Rev 14, 2021-2030.

55. Cransac-Miet A, Zeller M, Chagué F, et al. (2021) Impact of COVID-19 lockdown on lifestyle adherence in stay-at-home patients with chronic coronary syndromes: towards a time bomb. Int J Cardiol 323, 285-287.

56. Jia P, Zhang L, Yu W, et al. (2020) Impact of COVID-19 lockdown on activity patterns, weight status among youths in China: the COVID-19 Impact on Lifestyle Change Survey (COINLICS). Int J Obes $1-5$.

57. Malkawi SH, Almhdawi K, Jaber AF, et al. (2020) COVID-19 quarantine-related mental health symptoms and their correlates among mothers: a cross sectional study. Matern Child Health $J 1$.

58. de Matos DG, Aidar FJ, Almeida-Neto PF, et al. (2020) The impact of measures recommended by the government to limit the spread of coronavirus (COVID-19) on physical activity levels, quality of life, and mental health of Brazilians. Sustainability 12, 9072

59. Ruissen MM, Regeer H, Landstra CP, et al. (2021) Increased stress, weight gain, less exercise in relation to glycemic control in people with type 1, type 2 diabetes during the COVID-19 pandemic. BMJ Open Diab Res Care 9, e002035.

60. Ahmed HO (2020) The impact of social distancing and selfisolation in the last corona COVID-19 outbreak on the body weight in Sulaimani governorate-Kurdistan/Iraq, a prospective case series study. Ann Med Surg 59, 110-117.

61. Gentile A, Torales J, O'Higgins M, et al. (2020) Phone-based outpatients' follow-up in mental health centers during the COVID-19 quarantine. Int J Soc Psychiatr 0020764020 979732.

62. Romero-Blanco C, Rodríguez-Almagro J, Onieva-Zafra MD, et al. (2020) Sleep Pattern Changes in Nursing Students during the COVID-19 Lockdown. Int J Environ Res Public Health 17, 5222.

63. Dogas Z, Kalcina LL, Dodig IP, et al. (2020) The effect of COVID-19 lockdown on lifestyle and mood in Croatian general population: a cross-sectional study. Croatian Med J 61, 309.

64. Onmez A, Gamsizkan Z, Özdemir 叉, et al. (2020) The effect of COVID-19 lockdown on glycemic control in patients with type 2 diabetes mellitus in Turkey. Diabetes Metab Syndrome: Clin Res Rev 14, 1963-1966.

65. Karatas S, Yesim T \& Beysel S (2021) Impact of lockdown COVID-19 on metabolic control in type 2 diabetes mellitus and healthy people. Primary Care Diabetes (In the Press).

66. Mason TB, Barrington-Trimis J \& Leventhal AM (2021) Eating to cope with the COVID-19 pandemic and body weight change in young adults. J Adolesc Health 68, 277-283.

67. Blaszczyk-Bębenek E, Jagielski P, Boleslawska I, et al. (2020) Nutrition behaviors in Polish adults before, during covid-19 lockdown. Nutrients 12, 3084.

68. Huber BC, Steffen J, Schlichtiger J, et al. (2020) Altered nutrition behavior during COVID-19 pandemic lockdown in young adults. Eur J Nutr 1-10.

69. Newman E, O'Connor DB \& Conner M (2007) Daily hassles and eating behaviour: the role of cortisol reactivity status. Psychoneuroendocrinology 32, 125-132.

70. Jakubowicz D, Barnea M, Wainstein J, et al. (2013) High caloric intake at breakfast vs. dinner differentially influences weight loss of overweight and obese women. Obesity $\mathbf{2 1}$, 2504-2512. 
71. McNeill LH, Kreuter MW \& Subramanian SV (2006) Social environment and physical activity: a review of concepts and evidence. Soc Sci Med 63, 1011-1022.

72. Swinburn B, Egger G \& Raza F (1999) Dissecting obesogenic environments: the development and application of a framework for identifying and prioritizing environmental interventions for obesity. Prev Med 29, 563-570.

73. Gallè F, Sabella EA, Da Molin G, et al. (2020) Understanding Knowledge and Behaviors Related to CoViD-19 Epidemic in Italian Undergraduate Students: the EPICO Study. Int $J$ Environ Res Public Health 17, 3481.

74. Salute M della (2020) Covid-19, how to follow an appropriate and healthy lifestyle when staying at home. http://www. salute.gov.it/portale/nuovocoronavirus/dettaglioNotizieNuovo Coronavirus.jsp?lingua $=$ italiano\&menu $=$ notizie $\& p=$ dalmini stero\&id $=4421$ (accessed July 2020).

75. Almandoz JP, Xie L, Schellinger JN, et al. (2020) Impact of COVID-19 stay-at-home orders on weight-related behaviours among patients with obesity. Clin Obes 10, e12386.

76. Kivimäki M, Head J, Ferrie JE, et al. (2006) Work stress, weight gain and weight loss: evidence for bidirectional effects of job strain on body mass index in the Whitehall II study. Int J Obes 30, 982-987.

77. Serlachius A, Hamer M \& Wardle J (2007) Stress and weight change in university students in the United Kingdom. Physiol Behav 92, 548-553.

78. Razzoli M \& Bartolomucci A (2016) The Dichotomous effect of chronic stress on obesity. Trends Endocrinol Metab 27, 504-515.

79. Dallman MF (2010) Stress-induced obesity and the emotional nervous system. Trends Endocrinol Metab 21, 159-165.

80. Adam TC \& Epel ES (2007) Stress, eating and the reward system. Physiol Behav 91, 449-458.

81. O'Connor DB, Jones F, Conner M, et al. (2008) Effects of daily hassles and eating style on eating behavior. Health Psychol 27, S20-S31.

82. Heatherton TF \& Baumeister RF (1991) Binge eating as escape from self-awareness. Psychol Bull 110, 86-108.

83. Haddad C, Zakhour M, Bou Kheir M, et al. (2020) Association between eating behavior, quarantine/confinement stressors during the Coronavirus disease 2019 outbreak. J Eat Disord 8, 40. doi: 10.1186/s40337-020-00317-0

84. Khan MA, Shah SM, Shehab A, et al. (2019) Screen time and metabolic syndrome among expatriate adolescents in the United Arab Emirates. Diabetes Metab Syndrome: Clin Res Rev 13, 2565-2569.

85. Stults-Kolehmainen MA \& Sinha R (2014) The effects of stress on physical activity and exercise. Sports Med $\mathbf{4 4}$, $81-121$.

86. Seigel K, Broman J-E \& Hetta J (2002) Behavioral activation or inhibition during emotional stress-implications for exercise habits and emotional problems among young females. Nord J Psychiatry 56, 441-446.

87. Vlahov D, Galea S, Resnick H, et al. (2002) Increased use of cigarettes, alcohol, and marijuana among Manhattan, New York, residents after the September 11th terrorist attacks. Am J Epidemiol 155, 988-996.

88. Chiolero A, Faeh D, Paccaud F, et al. (2008) Consequences of smoking for body weight, body fat distribution, and insulin resistance. Am J Clin Nutr 87, 801-809.

89. Poelman MP, Gillebaart M, Schlinkert C, et al. (2020) Eating behavior and food purchases during the COVID-19 lockdown: a cross-sectional study among adults in the Netherlands. Appetite 157, 105002.

90. Ruiz-Roso MB, de Carvalho Padilha P, Mantilla-Escalante DC, et al. (2020) Covid-19 confinement, changes of adolescent's dietary trends in Italy, Spain, Chile, Colombia, Brazil. Nutrients 12, 1807.

91. Ferrer R \& Klein WM (2015) Risk perceptions and health behavior. Curr Opin Psychol 5, 85-89.

92. Raude J, Lecrique JM, Lasbeur L, et al. (2020) Determinants of preventive behaviors in response to the COVID-19 pandemic in France: comparing the sociocultural, psychosocial and social cognitive explanations. Front Psychol 11, 584500. doi: 10.3389/fpsyg. 2020.584500

93. Adams EL, Caccavale LJ, Smith D, et al. (2020) Food insecurity, the home food environment, and parent feeding practices in the era of COVID-19. Obesity 28, 2056-2063.

94. Micha R, Mannar V, Afshin A, et al. (2020) 2020 Global nutrition report: action on equity to end malnutrition. Development Initiatives. https://eprints.mdx.ac.uk/id/eprint/30645

95. UNSCN (2020) COVID-19 pandemic: the evolving impact on how people meet the food system. https://www.unscn.org/ en/news-events/recent-news?idnews $=2065$ (accessed January 2021).

96. WHO (2020) As more go hungry and malnutrition persists, achieving Zero Hunger by 2030 in doubt, UN report warns. https://www.who.int/news/item/13-07-2020-as-more-gohungry-and-malnutrition-persists-achieving-zero-hungerby-2030-in-doubt-un-report-warns (accessed February 2021).

97. United Nations World Food Programme (2020) WFP at a glance. https://www.wfp.org/stories/wfp-glance (accessed December 2020).

98. Paslakis G, Dimitropoulos G \& Katzman DK (2021) A call to action to address COVID-19-induced global food insecurity to prevent hunger, malnutrition, and eating pathology. Nutr Rev 79, 114-116.

99. Schanzenbach D \& Pitts A (2020) How much has food insecurity risen? Evidence from the Census Household Pulse Survey. Institute for Policy Research (IPR) Rapid Research Report. Northwestern Institute for Policy Research. https://www.ipr. northwestern.edu/news/2020/schanzenbach-household-pulsesurvey-analysis.html

100. El Zein A, Colby SE, Zhou W, et al. (2020) Food insecurity is associated with increased risk of obesity in US college students. Curr Dev Nutr $\mathbf{4}$, nzaa120.

101. Sheth J (2020) Impact of Covid-19 on consumer behavior: will the old habits return or die? J Bus Res 117, 280-283.

102. Mehta S, Saxena T \& Purohit N (2020) The new consumer behaviour paradigm amid COVID-19: permanent or transient? J Health Manag 22, 291-301.

103. Baraniuk C (2020) Fears grow of nutritional crisis in lockdown UK. BMJ 370, m3193.

104. Hajifathalian K, Kumar S, Newberry C, et al. (2020) Obesity is associated with worse outcomes in COVID-19: analysis of Early Data From New York City. Obesity 28, 1606-1612.

105. Ho FK, Celis-Morales CA, Gray SR, et al. (2020) Modifiable, non-modifiable risk factors for COVID-19: results from UK Biobank prospective cohort study. BMJ Open 10, e040402.

106. Huang Y, Yao LU, Huang Y-M, et al. (2020) Obesity in patients with COVID-19: a systematic review, meta-analysis. Metabolism 154378.

107. Hill MA, Sowers JR \& Mantzoros CS (2020) Commentary: COVID-19 and obesity pandemics converge into a syndemic requiring urgent and multidisciplinary action. Metabolism 114, 154408.

108. Eknoyan G (2006) A history of obesity, or how what was good became ugly and then bad. Adv Chronic Kidney Dis 13, 421-427.

109. Gagnier JJ, Moher D, Boon H, et al. (2012) Investigating clinical heterogeneity in systematic reviews: a methodologic review of guidance in the literature. BMC Med Res Method 12, 111. 